\title{
Regorafenib impairs mitochondrial functions, activates AMP-activated protein kinase, induces autophagy, and causes rat hepatocyte necrosis
}

Zuquan Weng ${ }^{1, *}$, Yong Luo ${ }^{1, *}$, Xi Yang ${ }^{1}$, James J. Greenhaw ${ }^{1}$, Haibo Li $^{1,2}$, Liming Xie ${ }^{1,3}$, William B. Mattes ${ }^{4}$, Qiang $\operatorname{Shi}^{1{ }^{1 * *}}$

${ }^{1}$ Division of Systems Biology, National Center for Toxicological Research, Food and

Drug Administration, 3900 NCTR Road, Jefferson, AR 72079, USA

${ }^{2}$ Present address: Department of Microbiology, Nantong Center for Disease Control and Prevention, 189 South

Gongnong Road, Nantong, Jiangsu, 226007, China

${ }^{3}$ Present address: Regulatory Science, University of Southern California, 1540 Alcazar Street, CHP 140, Los

Angeles, CA 90033, USA

${ }^{4}$ PharmPoint Consulting, 17014 Hersperger Lane, Poolesville, MD 20837, USA

* These authors contributed equally to this work.

** Corresponding author. E-mail address: qiang.shi@fda.hhs.gov Tel: 1-870-543-7365 Fax: 1-870-543-7736

Disclaimer: The information in these materials is not a formal dissemination of information by FDA and does not represent agency position or policy. Part of the data was presented as a poster at the 53rd Annual Meeting of Society of Toxicology in Phoenix, AZ, on March 25, 2014. 


\section{Acknowledgements}

This project is supported by the U.S. FDA's Office of Women's Health. Drs. Zuquan Weng and Haibo Li are supported by the Research Participation Program at the National Center for Toxicological Research administrated by the Oak Ridge Institute for Science and Education through an interagency agreement between the U.S. Department of Energy and the U.S. FDA. Dr. Haibo Li is also partially supported by the International Cooperation and Exchanges (2012) program from the Department of Health in Jiangsu Province, China. The authors would like to thank Dr. Donna L. Mendrick for her critical review of this manuscript. 


\title{
Regorafenib impairs mitochondrial functions, activates AMP-activated protein kinase, induces autophagy, and causes rat hepatocyte necrosis
}

\begin{abstract}
The tyrosine kinase inhibitor regorafenib was approved by regulatory agencies for cancer treatment, albeit with strong warnings of severe hepatotoxicity included in the product label. The basis of this toxicity is unknown; one possible mechanism, that of mitochondrial damage, was tested. In isolated rat liver mitochondria, regorafenib directly uncoupled oxidative phosphorylation (OXPHOS) and promoted calcium overload-induced swelling, which were respectively prevented by the recoupler 6-ketocholestanol (KC) and the mitochondrial permeability transition (MPT) pore blocker cyclosporine A (CsA). In primary hepatocytes, regorafenib uncoupled OXPHOS, disrupted mitochondrial inner membrane potential (MMP), and decreased cellular ATP at $1 \mathrm{~h}$, and triggered MPT at $3 \mathrm{~h}$, which was followed by necrosis but not apoptosis at $7 \mathrm{~h}$ and $24 \mathrm{~h}$, all of which were abrogated by KC. The combination of the glycolysis enhancer fructose plus the mitochondrial ATPase synthase inhibitor oligomycin A abolished regorafenib induced necrosis at $7 \mathrm{~h}$. This effect was not seen at $24 \mathrm{~h}$ nor with the fructose or oligomycin A separately. CsA in combination with trifluoperazine, both MPT blockers, showed similar effects. Two compensatory mechanisms, activation of AMP-activated protein kinase (AMPK) to ameliorate ATP shortage and induction of autophagy to remove dysfunctional mitochondria, were found to be mobilized. Hepatocyte necrosis was enhanced either by the AMPK inhibitor Compound $\mathrm{C}$ or the autophagy inhibitor chloroquine, while autophagy inducer rapamycin was strongly cytoprotective. Remarkably, all toxic effects were observed at clinically-relevant concentrations of 2.5 to $15 \mu \mathrm{M}$. These data suggest that uncoupling of OXPHOS and the resulting ATP shortage and MPT induction are the key mechanisms for regorafenib induced hepatocyte injury, and AMPK activation and autophagy induction serve as pro-survival pathways against such toxicity.
\end{abstract}

Keywords: regorafenib, hepatotoxicity, mitochondrion, rat, hepatocyte, necrosis 


\section{Introduction}

Regorafenib is a broad spectrum tyrosine kinase inhibitor (Davis et al. 2013). It was recently approved for treatment of metastatic colorectal cancer and advanced gastrointestinal stromal tumors by the U.S. Food and Drug Administration (FDA) (FDA 2012a), the Health Canada (Health-Canada 2013), and the European Medicines Agency (EMA) (EMA 2013). Based on clinical trial data, these regulatory agencies have called attention to liver toxicity as one of the most serious adverse effects of regorafenib (EMA 2013; FDA 2012a; Health-Canada 2013). A black box warning, the strongest warning about a drug's adverse effect issued by the FDA, has been included in the U.S. product labeling of regorafenib to highlight its liver risks. Similarly, the product monograph of regorafenib endorsed by Health Canada also contains black-boxed "serious warnings and precautions" about liver injuries, and the EMA requires that a "black inverted triangle" be displayed in the package leaflet to indicate that such risk is under "additional monitoring". As clinical trials are ongoing to examine if regorafenib provides an alternative treatment for hepatocellular carcinoma (Bruix et al. 2013), its clinical usage is expected to increase significantly, as are the risks of liver injury. Of note, studies conducted in preclinical test species identified the liver as a target organ of regorafenib toxicity in multiple animal species (mouse, rat and dog) (FDA 2012b), suggesting that animal based models may be used to understand the basis for regorafenib's clinical hepatotoxicity. However, the mechanism for regorafenib induced hepatotoxicity has not been explored to date, limiting the development of strategies to minimize liver risks.

Various mechanisms have been proposed as operative in drug hepatotoxicity. Among these, mitochondrial impairment appears to be commonly shared by many drugs and chemicals (Jones et al. 2010; Pessayre et al. 2012). Mitochondrial damage is usually considered as a drug's "off-target" effect contributing to adverse reactions, though recent evidence suggests that it may also be important for some drugs' pharmacological effects. For example, metformin, the first-line drug for type 2 diabetes, causes slight inhibition of mitochondrial respiratory complex I leading to downstream reactions responsible for the anti-diabetic and 
possible anti-cancer effects (Bridges et al. 2014; Pernicova and Korbonits 2014). As for drug induced liver injury (DILI), it has been long recognized that acetaminophen overdose induced hepatotoxicity was associated with inhibition of mitochondrial respiration (Meyers et al. 1988). Most recently, it was suggested that a battery of in vitro mitochondrial assays may be predictive of a chemical's potential to induce DILI in humans (Porceddu et al. 2012), though the need for very high concentrations of drugs to trigger positive mitochondrial effects casts doubt on its in vivo relevance. This study examined if regorafenib causes mitochondrial dysfunction in isolated rat liver mitochondria and primary cultured hepatocytes. At concentrations comparable to the human therapeutic exposure of $8 \mu \mathrm{M}$ in the circulation (FDA 2012b), regorafenib uncoupled hepatic mitochondrial oxidative phosphorylation (OXPHOS), dissipated mitochondrial inner membrane potential (MMP), caused cellular adenosine triphosphate (ATP) shortage, and later induced mitochondrial permeability transition (MPT), all of which eventually led to necrosis but not apoptosis in primary rat hepatocytes. At the same time AMP-activated protein kinase (AMPK), the master regulator of energy homeostasis, and autophagy, a process to remove damaged mitochondria, were activated to promote hepatocyte survival. Thus the induction of liver mitochondrial dysfunction does indeed seem to be the basis for regorafenib-induced hepatotoxicity.

\section{Materials and methods}

\subsection{Reagents and chemicals}

Regorafenib with a purity of $99.62 \%$ was obtained from Selleck Chemicals (Houston, TX; catalog number S1178; batch number S117804). Calcein acetoxymethyl ester (Calcein AM) and 5,5',6,6'-tetrachloro1,1',3,3'-tetraethylbenzimidazolocarbocyanine iodide (JC-1) were from Life Technologies Corporation (Grand Island, NY). CellTiter-Glo Luminescent Cell Viability Assay kit (for measuring cellular ATP levels) and Caspase-Glo® 3/7 Assay Systems (for measuring caspase 3/7 activity) were from Promega (Madison, WI). AMPK Activator III (DHPO) and Compound C (CAS 866405-64-3) were from EMD Millipore (Billerica, MA). All other chemicals were from Sigma-Aldrich (St. Louis, MO).

\subsection{Animal care}


Male Sprague-Dawley rats weighing 250 to $400 \mathrm{~g}$ were obtained from the U.S. Food and Drug Administration National Center for Toxicological Research (NCTR) breeding colonies. Animal care and experimental procedures were approved by the NCTR Institutional Animal Care and Use Committee in accordance with the National Institutes of Health (NIH) "Guide for the Care and Use of Laboratory Animals."

\subsection{Mitochondria isolation and drug treatment}

Rat liver mitochondria were isolated using a published procedure (Frezza et al. 2007). Only mitochondria with a respiratory control ratio $(\mathrm{RCR})>3$ were used and all experiments were completed within $5 \mathrm{~h}$ after isolation. The protein concentration was adjusted to $0.5 \mathrm{mg} / \mathrm{ml}$ using the respiration buffer (Frezza et al. 2007). Regorafenib was dissolved in DMSO and added to final concentrations of $0,1,2.5,5$, and $10 \mu \mathrm{M}$. The final DMSO concentration was $0.1 \%$. After 3 min incubation oxygen consumption was measured as described below. In specified cases, mitochondria were treated for 3 min with $2 \mu \mathrm{g} / \mathrm{ml}$ oligomycin A prior to addition of regorafenib, and after 3 min incubation $200 \mu \mathrm{M}$ 6-ketocholestanol (KC) was added to observe the recoupling effect (Starkov et al. 1997).

\subsection{Hepatocyte culture and drug treatment}

Primary hepatocytes were cultured in William's E medium which contains $10 \mathrm{mM}$ D-glucose as detailed previously (Shi et al. 2011). Briefly, rats were anesthetized by sodium pentobarbital and the liver was perfused in situ with a buffer containing $137 \mathrm{mM} \mathrm{NaCl}, 5.4 \mathrm{mM} \mathrm{KCl}, 0.5 \mathrm{mM} \mathrm{KH} \mathrm{PO}_{4}, 0.6 \mathrm{mM} \mathrm{Na}_{2} \mathrm{PO}_{4}$, $25 \mathrm{mM} \mathrm{NaHCO}{ }_{3}, 5 \mathrm{mM}$ glucose, $5 \mu \mathrm{g} / \mathrm{ml}$ bovine insulin, $50 \mathrm{unit} / \mathrm{ml}$ penicillin and $50 \mu \mathrm{g} / \mathrm{ml}$ streptomycin for about 8 min. Then $2 \mathrm{mM} \mathrm{CaCl}_{2}, 0.03 \%$ collagenase (Sigma catalog number C5138), and $0.01 \%$ soybean trypsin inhibitor was added to the perfusion buffer and the liver was perfused for additional $5 \mathrm{~min}$. The digested liver was gently homogenized to release the cells and an isotonic Percoll@ solution (pH7.3) was used to purify the hepatocytes (Shi et al. 2011). Of note, our preliminary data show that hepatocytes from male and female rats had the similar response to regorafenib. Therefore only male rats were used in subsequent experiments. The cells were allowed to attach overnight before drug treatment. Regorafenib was added to culture medium at final concentrations of $0,2.5,5,7.5,10$ and $15 \mu \mathrm{M}$. The final DMSO 
concentration was $0.1 \%$. In specified cases, cells were treated with regorafenib in the presence of either cyclosporine A (CsA; $0.5 \mu \mathrm{M}$ ) plus trifluoperazine (TFP; $2.5 \mu \mathrm{M})$ (Imberti et al. 1993; Reid et al. 2005), or fructose $(15 \mathrm{mM})$, or fructose plus oligomycin A $(0.6 \mathrm{ng} / \mathrm{ml})$ (Nieminen et al. 1994). To inhibit autophagy, cells were treated with $15 \mu \mathrm{M}$ chloroquine (CQ). To induce autophagy, $5 \mu \mathrm{M}$ rapamycin was used (Ni et al. 2012). Compound $\mathrm{C}(10 \mu \mathrm{M})$ was used to inhibit AMPK activation (Saberi et al. 2014). A previously published procedure (Sunakawa et al. 2013) was modified to collect cells for measuring oxygen consumption. Briefly, hepatocytes seeded in $100 \times 20 \mathrm{~mm}$ dishes were washed by PBS and detached by trypsinization. Cells were re-suspended in respiration buffer at $2 \times 10^{6}$ cell $/ \mathrm{ml}$ and $0.0075 \%$ digitonin was added for 3 min to permeablize the cell membrane (Sunakawa et al. 2013). The stock solution of 7.5\% digitonin in water was prepared by heating at $95^{\circ} \mathrm{C}$ for $5 \mathrm{~min}$ immediately before use.

\subsection{Mitochondrial oxygen consumption}

Clark type oxygen electrodes were used to measure oxygen consumption. Two Oxytherm systems from the Hansatech Instruments Ltd were used. Each mitochondrial sample (1 mg/ml, $2 \mathrm{ml})$ or preparation of permeabilized hepatocytes $\left(2 \times 10^{6} \mathrm{cell} / \mathrm{ml}, 2 \mathrm{ml}\right)$ was evenly divided, with one half used to measure glutamate-malate driven respiration and the other succinate driven respiration; the two measurements for the same sample were thus taken simultaneously. The succinate concentration was $5 \mathrm{mM}$, and the glutamate/malate concentrations were $5 \mathrm{mM}$ and $2.5 \mathrm{mM}$, respectively, while for measuring oligomycin Ainhibited respiration, the concentrations of these substrates were decreased to 0.25 -fold the original level so that the oxygen in the reaction tubes would not be exhausted too quickly. The ADP concentration was 100 $\mu \mathrm{M}$ and $300 \mu \mathrm{M}$ for mitochondria and permeabilized hepatocytes, respectively. The maximal oxygen consumption rate after addition of ADP was assigned as state 3 respiration. When the added ADP is converted to ATP, the oxygen consumption rate decreases significantly to a slower rate, which was assigned as state 4 respiration. The RCR was calculated by dividing the state 3 respiration rate by the state 4 respiration rate. The well-established uncoupler carbonyl cyanide m-chlorophenyl hydrazone (CCCP) was used as a control.

\subsection{Mitochondria swelling assay}


A published procedure (Waldmeier et al. 2002) with only slight modifications was used to measure mitochondria swelling. Briefly, freshly isolated rat liver mitochondria (1 $\mathrm{mg} / \mathrm{ml}$ protein) were re-suspended in a buffer containing $70 \mathrm{mM}$ sucrose, $190 \mathrm{mM}$ mannitol, $20 \mathrm{mM}$ HEPES, $5 \mathrm{mM}$ glutamate, $0.5 \mathrm{mM}$ malate ( $\mathrm{pH}$ 7.5), and then regorafenib was added to final concentrations of 1 to $15 \mu \mathrm{M}$, with DMSO $(0.1 \%)$ used as a control. After $3 \mathrm{~min}, 1 \mathrm{mM} \mathrm{K} \mathrm{HPO}_{4}$ and $25 \mu \mathrm{M} \mathrm{CaCl}_{2}$ were added, and then the decrease in absorbance at $535 \mathrm{~nm}$ was immediately measured using a Synergy 2 Multi-Mode Microplate Reader every $2 \mathrm{~min}$ for $30 \mathrm{~min}$. To prevent MPT, CsA $(1 \mu \mathrm{M})$ was added for $1 \mathrm{~min}$ prior to regorafenib treatment.

\subsection{MMP detection}

Hepatocytes cultured in 6-well plates were trypsinized and re-suspended at $0.6 \times 10^{6} \mathrm{cell} / \mathrm{ml}$ in culture medium containing $10 \mu \mathrm{M} \mathrm{JC}-1$. Cells were incubated at $37^{\circ} \mathrm{C}$ for $15 \mathrm{~min}$, with occasional shaking to ensure a good suspension. Cells were pelleted by centrifugation and re-suspended at $1.2 \times 10^{6} \mathrm{cell} / \mathrm{ml}$ in PBS. A small fraction of the cells (usually $20 \mu \mathrm{l}$ ) was mounted on a microscope slide to observe fluorescence change using the FITC and the Texas Red channels, and $50 \mu 1$ cells were transferred into a 96-well black plate for the measurement of JC-1 fluorescence by a plate reader. The excitation wavelength was $488 \mathrm{~nm}$, and the emission wavelengths were $530 \mathrm{~nm}$ and $590 \mathrm{~nm}$. The fluorescence intensity ratio between $590 \mathrm{~nm}$ and $530 \mathrm{~nm}$ was calculated to reflect changes in MMP (Cervinkova et al. 2007).

\subsection{MPT detection}

Calcein AM with cobalt (II) chloride quenching was used for MPT detection. While calcein AM readily enters cells and universally stains all the mitochondrial, nuclear and cytosolic compartments, cobalt (II) chloride selectively quenches the fluorescence in the latter two subcellular locations, leaving the signal in mitochondria intact. When MPT occurs, the mitochondrion traps less calcein AM and cellular fluorescence is therefore decreased (Petronilli et al. 1999). A published procedure using calcein AM (Petronilli et al. 1999) was modified to monitor MPT in rat hepatocytes. Briefly, hepatocytes seeded in 6-well plates were trypsinized and re-suspended at $1 \times 10^{6} \mathrm{cell} / \mathrm{ml}$ in modified Hanks' Balanced Salt Solution (HBSS) containing $0.2 \mu \mathrm{M}$ calcein AM. Cells were incubated at $37^{\circ} \mathrm{C}$ with occasional shaking for $10 \mathrm{~min}$, and then $400 \mu \mathrm{M}$ cobalt (II) chloride was added. After $1 \mathrm{~min}$, cells were pelleted by centrifugation and re-suspended 
in PBS after washing with HBSS to remove excessive calcein AM and cobalt (II) chloride. All the processed samples were immediately analyzed on an Aria-III flow cytometer (BD Biosciences) using the $488 \mathrm{~nm}$ laser and FITC detection channel. The flow cytometry data were analyzed using FACS DIVA7 software (BD Biosciences). Mean immunofluorescence intensity (MFI) value was used to determine the average staining intensity of calcein AM.

\subsection{ATP detection and lactate dehydrogenase (LDH) leakage assay}

Cellular ATP levels were determined using a commercial kit as described previously (Sonko et al. 2011). The LDH leakage assay, whose results correlated well with the trypan blue exclusion method, was performed following the published procedure (Shi et al. 2011).

\subsection{Apoptosis detection}

Hepatocytes were grown in 6-well plates on $22 \times 22 \mathrm{~mm}$ cover glasses coated with type I bovine collagen. Apoptotic cells were immediately detected by Nikon Eclipse Ti microscopy at the $405 \mathrm{~nm}$ channel following staining for $30 \mathrm{~min}$ in culture medium containing $8 \mu \mathrm{g} / \mathrm{ml}$ Hoechst 33258. Cells displaying nuclear condensation and chromatin fragmentation were considered as apoptotic. At least 500 cells were counted (Nagai et al. 2002). Apoptosis was also determined by measuring caspase 3/7 activity (CaspaseGlo® 3/7 Assay) following the manufacturer's protocol. Briefly, hepatocytes were seeded in coated 96well white plates with a clear bottom, and incubated with regorafenib for 1 to $24 \mathrm{~h}$. Cells were washed once with PBS, after which an equal volume of culture medium and caspase 3/7 substrates ( $50 \mu 1$ for each) were added. After incubation at $37^{\circ} \mathrm{C}$ for $30 \mathrm{~min}$, cellular luminescence was measured to determine caspase $3 / 7$ activity. The luminescence signal was normalized to DMSO (0.1\%) treated cells. Diclofenac (400 $\mu \mathrm{M})$ was used as a positive control to induce hepatocytes apoptosis (Gomez-Lechon et al. 2003).

\subsection{Detection of AMPK activation and autophagy induction}

The previously published procedures were modified to detect AMPK and autophagy activation (Ding and Yin 2009; Lim et al. 2012). Briefly, cells were lysed using an ice-cold buffer containing $50 \mathrm{mM}$ Tris-HCl (pH 7.4), $50 \mathrm{mM}$ sodium fluoride, $5 \mathrm{mM}$ sodium pyrophosphate, $5 \mathrm{mM}$ sodium orthovanadate, $1 \%$ sodium dodecyl sulfate (SDS), $0.25 \%$ sodium deoxycholate, $1 \%$ Triton X-100, 1 mM phenylmethanesulfonyl 
fluoride, $1 \mathrm{mM}$ benzamidine, $5 \mu \mathrm{g} / \mathrm{ml}$ soybean trypsin inhibitor, 1:100 protease inhibitor cocktail (SigmaAldrich, St. Louis, MO; catalog number P8340), 2 mM ethylenediaminetetraacetic acid (EDTA) and $2 \mathrm{mM}$ ethylene glycol tetraacetic acid (EGTA). The lysates were centrifuged at 20,000 g for $10 \mathrm{~min}$ and then the supernatant was used for Western blot. AMPK activation was detected using an antibody against phosphoAMPK alpha (threonine-172) (p-AMPK) (Cell Signaling Technology, Beverly, MA; catalog number 8359S) (Lim et al. 2012). An AMPK antibody (Cell Signaling Technology, Beverly, MA; catalog number 8359S) was also used to determine AMPK levels. The antibody against the microtubule-associated protein 1 light chain 3 (LC3-II) (Novus Biologicals, Littleton, CO; catalog number NB100-2220) was used to assess autophagy (Ding and Yin 2009). The same samples were also probed with a beta-actin antibody (Santa Cruz Biotechnology, Dallas, TX; catalog number sc-1616) for data normalization.

\subsection{Statistical analysis}

One-way analysis of variance (ANOVA) followed by Dunnett's test were used to compare the means among groups using the Graph Pad Prism software (version 6; La Jolla, CA). A $p$ value of less than 0.05 or 0.01 was considered to be of statistical significance.

\section{Results}

\subsection{Regorafenib directly uncoupled liver mitochondrial respiration}

Freshly isolated rat liver mitochondria were used to observe the direct effect of regorafenib on mitochondrial respiration. Fig. 1A shows that the glutamate/malate-driven state 4 respiration was significantly increased by regorafenib in a concentration-dependent manner, with a maximal increase of approximately 7 -fold the base line level at 5 and $10 \mu \mathrm{M}$, and the state 3 respiration was also enhanced, though to a much less extent, that is, about 2 -fold the base line level at 5 and $10 \mu \mathrm{M}$. The corresponding RCR began to decrease at $1 \mu \mathrm{M}$ regorafenib and reached about 1 that is indicative of complete uncoupling at 2.5 to $10 \mu \mathrm{M}$. Very similar results were obtained with succinate-driven respiration (Fig. 1B), though in this case the maximal stimulation of the state 4 respiration was approximately 3 -fold and the state 3 respiration was unaffected. The effects of regorafenib were similar to the classical uncoupler CCCP. These data provide initial evidence that regorafenib is an uncoupler of mitochondrial respiration. 
To confirm that regorafenib is an uncoupler, oligomycin A was used to eliminate the possible confounding effect of ADP on oxygen consumption (Brand and Nicholls 2011). It was found that oligomycin Ainhibited respiration, whether it was driven by glutamate/malate (Fig. 1C) or succinate (Fig. 1D), was concentration-dependently enhanced by regorafenib, with a maximal stimulation of about 3 -fold at $10 \mu \mathrm{M}$. These data provide convincing evidence that regorafenib uncouples mitochondrial respiration.

To further characterize regorafenib-induced uncoupling, the well-established recoupler 6-ketocholestanol (KC) (Starkov et al. 1997) was used. Fig. 1C and 1D show that regorafenib-induced stimulation of oxygen consumption was significantly diminished, but not completely prevented, by $200 \mu \mathrm{M} \mathrm{KC}$, indicating that regorafenib uncouples mitochondrial respiration partially by mechanisms similar to classical uncouplers such as carbonyl cyanide-4-(trifluoromethoxy)phenylhydrazone (FCCP) (Starkov et al. 1997). KC was only effective against FCCP or CCCP induced uncoupling, but had no effects on fatty acids induced uncoupling, possibly due to the involvement of thermogenin and ATP/ADP-antiporter in the latter case (Starkov et al. 1997). Our data suggest that regorafenib may uncouple respiration by mechanisms involved in both FCCP and fatty acids induced uncoupling.

\subsection{Regorafenib promoted calcium overload-induced mitochondrial swelling}

A mitochondrial swelling assay, which may reflect MPT but does not directly measure it (Waldmeier et al. 2002), was used to test regorafenib effects on MPT in isolated mitochondria. Fig. 1E shows that regorafenib at 2.5 to $15 \mu \mathrm{M}$, but not $1 \mu \mathrm{M}$, remarkably enhanced calcium overload-induced mitochondrial swelling in a concentration-dependent fashion, and Fig. $1 \mathrm{~F}$ shows that when mitochondria were pretreated with CsA, a cyclophilin D protein (CypD) binding drug (Waldmeier et al. 2002), the swelling triggered by regorafenib was completely prevented. These data indicate that regorafenib induces MPT when calcium homeostasis is disrupted.

\subsection{Regorafenib uncoupled mitochondrial respiration in primary hepatocytes}

To observe if regorafenib uncouples mitochondrial respiration in intact cells, rat hepatocytes were treated for $1 \mathrm{~h}$ and mitochondrial functions determined. Of note, hepatocyte death measured by LDH leakage (described later in Fig. 3) or caspase activation (data not shown) did not occur at this time point. Fig. 2 
shows that both glutamate/malate (Fig. 2A) and succinate (Fig. 2B) driven respiration were significantly uncoupled, as demonstrated by a remarkably increase in the state 4 respiration and a sharp decrease in RCRs. In the meantime, the state 3 respiration was barely affected. A near complete uncoupling, as determined by an RCR of about 1 , was observed at $15 \mu \mathrm{M}$ regorafenib. The stimulation of the state 4 respiration and the decrease in RCR was significantly antagonized by the recoupler KC, particularly in the 5 to $10 \mu \mathrm{M}$ groups, for which the uncoupling effect of regorafenib was almost completely abrogated. However, the uncoupling induced by $15 \mu \mathrm{M}$ regorafenib was only partially abolished by KC (Fig. 2A and 2B). These data demonstrate that regorafenib induces $\mathrm{KC}$-preventable uncoupling in primary hepatocytes.

\subsection{Regorafenib decreased MMP in primary hepatocytes}

As uncouplers may disrupt MMP (Brand and Nicholls 2011), the effect of regorafenib on hepatocyte MMP was determined using JC-1 at $1 \mathrm{~h}$. JC-1 forms aggregates (which show red fluorescence) in cells with normal MMP and exists as monomers (which show green fluorescence) when MMP is disrupted (Pernelle et al. 2011). Fig. 2C shows that hepatocytes treated with vehicle (DMSO) were stained predominantly red with almost no green signal, while regorafenib treated cells displayed a concentration-dependent decrease in red signal and a corresponding increase in green signal, indicating that MMP was depolarized. Again this detrimental effect was significantly ameliorated by the recoupler KC. The quantitative measurement of the red vs. green fluorescence ratio is presented in Fig. 2D, which confirmed that MMP was depolarized after regorafenib treatment, and the recoupler KC profoundly alleviated this effect. These data suggest that regorafenib disrupted MMP via its uncoupling effects.

\subsection{Regorafenib triggered MPT in primary hepatocytes}

As Fig. 1E and 1F suggest that regorafenib may directly interact with one of the mPTP component CypD in isolated mitochondria, we next examined if regorafenib induces MPT in primary hepatocytes. A representative flow cytometer measurement is shown in Fig. 2E, and the mean and SD of three independent experiments are shown Fig. 2F. Both figures show that the calcein AM fluorescence signal in mitochondria after $3 \mathrm{~h}$ started to decrease at the lowest dose of regorafenib $(2.5 \mu \mathrm{M})$, and the decrease became statistically significant at 5 to $15 \mu \mathrm{M}$, indicating that regorafenib induced MPT in hepatocytes at higher 
concentrations. Notably, the MPT signal was only about $15 \%$ of the control in the $15 \mu \mathrm{M}$ regorafenib group. No changes were observed at $1 \mathrm{~h}$ or $2 \mathrm{~h}$ (data not shown) when significant uncoupling already commenced (Fig. 2A-B), indicating that MPT was a downstream effect of mitochondrial uncoupling. This notion was further strengthened by the observation that the recoupler KC remarkably prevented MPT (Fig. 2E and 2F). Specifically, the MPT induced by regorafenib doses of 2.5 to $7.5 \mu \mathrm{M}$ was almost completely prevented by KC. These data provide strong evidence that regorafenib induces MPT at a later time point via its initial uncoupling effects.

\subsection{Regorafenib caused ATP shortage in primary hepatocytes}

Severe mitochondrial uncoupling could cause cellular ATP shortage in hepatocytes (Masubuchi et al. 2000). Fig. 3A-D show that regorafenib indeed lowered cellular ATP in a time- and concentration-dependent manner. It can be seen that ATP began to decrease as early as $1 \mathrm{~h}$ when no LDH leakage was observed, and such decrease was completely prevented by the recoupler KC (Fig. 3A), suggesting that ATP shortage was due to the uncoupling effect of regorafenib. At $3 \mathrm{~h}$, ATP levels continued to drop, and KC only partially prevented such decrease at $15 \mu \mathrm{M}$ regorafenib (Fig. 3B), which is in line with the finding in Fig. 2A-B that $\mathrm{KC}$ only partially prevented regorafenib's uncoupling effect at high concentrations. A similar trend was observed at $7 \mathrm{~h}$ and $24 \mathrm{~h}$ when LDH leakage became apparent (Fig. 3C-D). Notably, $15 \mu \mathrm{M}$ regorafenib reduced cellular ATP by about $80 \%$ at $7 \mathrm{~h}$ when only about $25 \%$ cells were dead as determined by LDH release (Fig. 3C). These data demonstrate that ATP shortage, which is largely due to uncoupling, is an early event prior to cell death.

\subsection{Regorafenib induced necrosis but not apoptosis in hepatocytes}

Significant mitochondrial impairments, particularly MPT, will eventually lead to hepatocyte death, which can be either apoptotic or necrotic (Malhi et al. 2010). Two methods were used to detect apoptosis, nuclear fragmentation/chromatin condensation and caspase 3/7 activity. As a positive control, the well-established apoptosis-inducing drug diclofenac (Gomez-Lechon et al. 2003) was tested in parallel with regorafenib. Fig $3 \mathrm{E}$ shows that regorafenib did not induce nuclear condensation or chromatin fragmentation at $7 \mathrm{~h}$, both of which were readily observed in diclofenac-treated cells, as indicated by arrows. The quantitative results of 
Fig. 3E were shown in Fig. 3F. Fig. 3G shows that regorafenib at $7 \mathrm{~h}$ also did not change caspase 3/7 activities, which were remarkably increased by diclofenac. These results demonstrate that regorafenib did not induce hepatocyte apoptosis at $7 \mathrm{~h}$. Of note, a similar trend was obtained at $3 \mathrm{~h}$ and $24 \mathrm{~h}$ (data not shown).

Hepatocyte necrosis was quantified by LDH leakage, which reflects cell membrane integrity and is a wellaccepted indicator of necrosis (Chan et al. 2013). Fig. 3A-B shows that no necrosis was observed at $1 \mathrm{~h}$ and $3 \mathrm{~h}$. A moderate but statistically meaningful increase of LDH release was seen at 10 and $15 \mu \mathrm{M}$ regorafenib at $7 \mathrm{~h}$ (Fig. 3C), which was significantly enhanced at $24 \mathrm{~h}$ (Fig. 3D), when $15 \mu \mathrm{M}$ regorafenib caused almost $100 \%$ cell death, and $7.5 \mu \mathrm{M}$ regorafenib led to about $20 \%$ cell death, while the lowest concentration of $2.5 \mu \mathrm{M}$ elicited only a negligible increase of LDH that was not statistically significant (Fig. 3D). Interestingly, the recoupler $\mathrm{KC}$ remarkably reduced regorafenib induced $\mathrm{LDH}$ release (Fig. 3C and 3D). These data suggest that regorafenib causes time- and concentration- dependent hepatocyte necrosis that is largely due to the uncoupling of mitochondrial respiration.

\subsection{Regorafenib induced necrosis was delayed by the glycolysis enhancer plus mitochondrial ATPase synthase inhibitor}

As cellular ATP depletion occurred prior to hepatocyte necrosis and enhanced ATP levels are associated with higher hepatocyte viability (Fig. 3), the effects of the glycolysis enhancer fructose, which provides an alternative energy source to mitochondrial OXPHOS, and the mitochondrial ATPase synthase inhibitor oligomycin A, which prevents the enhanced hydrolysis of ATP induced by uncoupling (Imberti et al. 1993; Masubuchi et al. 2000), were investigated. Fig. 4A shows that fructose alone was not effective in preventing regorafenib induced LDH release, but its combination with oligomycin A almost completely abolished regorafenib toxicity at $7 \mathrm{~h}$, indicating a causal relationship existed between uncoupling of OXPHOS and regorafenib induced hepatocyte death. Of note, oligomycin A alone was strongly toxic (date not shown). At $24 \mathrm{~h}$ (Fig. 4B), fructose or its combination with oligomycin A afforded no protection, likely because glycolysis alone failed to provide sufficient energy for survival or other pro-death pathways predominated. 


\subsection{Regorafenib induced necrosis was partially prevented by MPT blockers}

As MPT may determine if cells will survive or die (Malhi et al. 2010), the effect of MPT blockers CsA and TFP (CsA/TFP) (Kon et al. 2004) on regorafenib induced necrosis was investigated. As shown in Fig. 4C, hepatocyte death induced by $15 \mu \mathrm{M}$ regorafenib was partially decreased by CsA/TFP at $7 \mathrm{~h}$, indicating that MPT is causatively associated with regorafenib induced hepatocyte injury at the early stage. However, Fig. 4D shows that CsA/TFP afforded no protection at $24 \mathrm{~h}$, likely because regorafenib triggered other prodeath pathways (such as profound ATP depletion as shown in Fig. 3) that cannot be antagonized by MPT blockage after a relatively longer period of time.

\subsection{Regorafenib caused AMPK activation that might be cytoprotective}

As ATP shortage usually leads to AMPK activation to counterbalance energy deficiency (Viollet et al. 2009), the next set of experiments was carried out to observe if regorafenib activates AMPK in hepatocytes. As shown in Fig. 5A and 5B, pAMPK levels showed no changes at $1 \mathrm{~h}$, but were significantly enhanced at $3 \mathrm{~h}$ when no cell death occurred, and a further increase was seen at $7 \mathrm{~h}$, with a maximal increase of about 3 fold the control level. These data demonstrate that regorafenib activated AMPK pathway in hepatocytes.

Chemical regulators of AMPK were then used to examine if AMPK activation contributes to hepatocyte necrosis. Fig. 5C shows that the AMPK inhibitor Compound $\mathrm{C}$ barely affected regorafenib induced LDH release at $7 \mathrm{~h}$, but remarkably enhanced 7.5 and $10 \mu \mathrm{M}$ regorafenib induced cell death at $24 \mathrm{~h}$ (Fig. 5D), indicating that AMPK activation may serve as a pro-survival mechanism. Of note, the AMPK Activator III, DHPO, failed to reduce regorafenib induced hepatocyte necrosis (data not shown), very likely because the AMPK pathway was already maximally activated by regorafenib.

To observe if AMPK activation was triggered by the uncoupling effect of regorafenib, the recoupler KC was used. As shown in Fig. 5E and 5F, pAMPK levels at $7 \mathrm{~h}$ were significantly lower in regorafenib (5-10 $\mu \mathrm{M})$ plus $\mathrm{KC}$ treated hepatocytes than in regorafenib $(5-10 \mu \mathrm{M})$ alone treated cells, and $\mathrm{KC}$ alone showed no effects on pAMPK levels, suggesting that AMPK activation was very likely initiated by the uncoupling effect of regorafenib.

3.11. Regorafenib caused mild autophagy induction that alleviated cell death 
One well-established downstream effect of AMPK activation is autophagy induction (Egan et al. 2011), and MPT may also promote autophagy (Rodriguez-Enriquez et al. 2004), which can be either beneficial or detrimental to hepatocytes (Apostolova et al. 2011; Ni et al. 2012; Saberi et al. 2014). The last experiment then examined if autophagy plays a role in regorafenib induced hepatocyte injury. Fig. 6A and 6B show that high concentrations of regorafenib caused a mild accumulation of LC3-II at $7 \mathrm{~h}$, but not at $1 \mathrm{~h}$ or $3 \mathrm{~h}$, suggesting that autophagy was only slightly enhanced at later stages. Fig. 5C shows that the autophagy inducer rapamycin remarkably reduced regorafenib cytotoxicity, and the autophagy inhibitor chloroquine significantly enhanced regorafenib toxicity, indicating that the mild induction of autophagy by regorafenib is likely a protective mechanism to maintain cell viability.

\section{Discussion}

This is the first study to explore the mechanism of regorafenib hepatotoxicity. A schematic diagram of major findings is presented in Fig. 7.

Hepatotoxic drugs may damage liver mitochondria in many different ways, which can be interrelated (Jones et al. 2010; Pessayre et al. 2012). Here it was found that regorafenib uncoupled OXPHOS, depolarized MMP, depleted ATP, and triggered MPT. Experiments with the recoupler KC established that uncoupling of OXPHOS is the initiating event leading to downstream effects. Time-dependent studies show that uncoupling of OXPHOS occurred prior to MPT induction in primary hepatocytes (Fig. 2), further indicating that MPT is a consequence of uncoupling. Of note, both regulated and un-regulated MPT have been reported for other drugs (Okuda et al. 2010), and here it is clearly demonstrated that regorafenib induced MPT was regulated by CsA, which opens up the possibility of pharmacologically manipulating MPT to alleviate regorafenib toxicity. Interestingly, the mode of regorafenib induced hepatocyte death was predominantly necrosis but not apoptosis, very likely due to the lack of sufficient cellular ATP, which is a consequence of uncoupling, to help execute apoptosis (Malhi et al. 2006). This is also in line with other uncouplers such as usnic acid causing necrosis but not apoptosis in primary hepatocytes (Han et al. 2004).

We found that the rate of state 3 respirations was increased by regorafenib in isolated mitochondria (Fig 1), but not in primary hepatocytes (Fig. 2). The likely reason is that the secondary effects of uncoupling, such 
as the acidifying of cytosolic compartment and the impairment of functions of other organelles, may render the maximal respiration rate in cells "considerably less than" in isolated mitochondria (Brand and Nicholls 2011). Additional reasons may also include (1) isolated mitochondria were treated for only $3 \mathrm{~min}$, while the hepatocytes were treated for $1 \mathrm{~h}$ (2) digitonin was included to measure respirations in hepatocytes but not isolated mitochondria and (3) regorafenib may undergo extensive metabolism in hepatocytes producing metabolites that inhibit respiration. To explore the role of biotransformation in regorafenib induced mitotoxicity and cytotoxicity may be an interesting future direction.

Many drugs and chemicals have been recognized as uncouplers of liver mitochondrial respiration (Pessayre et al. 2012; Porceddu et al. 2012), but no therapeutic approaches abrogating this toxicity are currently available. Though fructose plus oligomycin A prevented uncoupler-induced cell death for a short period of time (Fig. 4A), they are of no clinical value because toxicity was unaffected at a longer treatment time (Fig. 4B), and oligomycin A is highly toxic by itself. The findings that the recoupler $\mathrm{KC}$ significantly antagonized regorafenib's uncoupling effect and therefore was protective against hepatocyte death is also of little clinical value, because the safety profiles of $\mathrm{KC}$ are unknown and we found that $\mathrm{KC}$ was strongly toxic to hepatocytes at higher concentrations of $\geq 200 \mu \mathrm{M}$ (data not shown).

Given that therapeutic agents to directly remedy uncoupling of OXPHOS are lacking, it becomes imperative to identify downstream pathways that contribute to regorafenib induced hepatocyte necrosis. Toward this end, we have found that MPT, AMPK, and autophagy were all associated with regorafenib cytotoxicity. Importantly, it is shown that MPT blockers and autophagy inducers, though exerting their effects at different levels in the signaling cascade, were both effective in reducing regorafenib cytotoxicity. As these protective agents are approved drugs that are in current clinical use, they might have potential to alleviate regorafenib hepatotoxicity. However, the "off-target" effects of these agents, such as the nephrotoxicity of CsA, which was originally approved as an immunosuppressant (Horina et al. 1993), must be taken into considerations for this purpose.

The mitotoxicity of regorafenib seems unlikely to be due to overall kinase inhibition, as several other kinase inhibitors have been shown to have no mitochondrial liability at a wide range of concentrations (Will et al. 2008). The fact that some approved kinase inhibitors are non-hepatotoxic (Shah et al. 2013) 
further indicates that regorafenib's hepatocyte toxicity is likely independent of overall kinase inhibition. On the other hand, the observation that AMPK was activated in response to regorafenib's mitotoxicity may very well contribute to its anti-cancer effects, because pharmacologically induced AMPK activation is tumor suppressive in both animal models and clinical trials (Shackelford and Shaw 2009). This is reminiscent of the recent finding that the drug metformin has anti-cancer properties due to its activation of AMPK by impairing mitochondrial OXPHOS (Leone et al. 2014). Future investigation will examine if regorafenib's mitotoxicity is involved in its pharmacological effect.

One interesting finding is that regorafenib caused mitotoxicity and subsequent cell death in hepatocytes at clinically-relevant concentrations. This is in contrast to the majority of previous reports on other drugs, for which detrimental mitochondrial effects were usually observed at exceptionally high drug concentrations, that is, up to ten-fold or even one hundred-fold of the average maximal blood concentration (Cmax), leaving the in vivo relevance uncertain (Dykens and Will 2007; Porceddu et al. 2012). At the recommended oral dose of $160 \mathrm{mg}$ per day, the average human Cmax of regorafenib was $8 \mu \mathrm{M}$ (FDA 2012b). At the human-equivalent dose, the average rat Cmax of regorafenib was $10 \mu \mathrm{M}$ (FDA 2012b). A high-fat meal or co-administration with a cytochrome P450 (CYPs) inhibitor increased the systemic exposure of regorafenib by 30-50\% in humans (FDA 2012b). It is thus clear that the Cmax in either rats or human could be as high as 12 to $15 \mu \mathrm{M}$. Here it was found that regorafenib impaired mitochondrial functions and caused hepatocyte necrosis at 5-15 $\mu \mathrm{M}$, concentrations readily achieved under in vivo conditions in both animals and humans, indicating that these in vitro results are very likely of in vivo relevance.

The finding of mitochondrial toxicity at in vitro regorafenib concentrations similar to clinically therapeutic blood concentrations begs the question as to why hepatotoxicity is not more frequently seen in preclinical studies or in patients treated with this drug. The Cmax in clinical studies was reached about $3 \mathrm{~h}$ after regorafenib administration, after which regorafenib blood concentration began to decrease rapidly $(60 \%$ Cmax at 8 h) (FDA 2012b), indicating that on average hepatocyte exposures to significant drug concentrations were limited. Our data show no cell death following $7 \mathrm{~h}$ exposures to $7.5 \mu \mathrm{M}$ regorafenib (though ATP was decreased by about 40\%). Taking into consideration that the liver has very strong capacity to regenerate, our data are consistent with the observation that most patients and animals show no 
apparent hepatotoxicity. Thus, the infrequent but profound hepatotoxicity seen in clinical settings may reflect individual variations in regorafenib pharmacokinetics such that in vivo hepatocyte exposures indeed approach the hepatotoxic level observed in the current experiments.

Our findings that regorafenib impairs mitochondrial functions may warrant further investigations to discover circulating biomarkers leaked from damaged liver mitochondria to better monitor regorafenib hepatotoxicity, as has been recently reported for acetaminophen overdose in humans (McGill et al. 2012). It may also warrant future structural modifications on regorafenib to reduce or eliminate mitotoxicity while retaining its pharmacological effects.

This study also suggests that when regorafenib is co-administrated with drugs known to impair mitochondrial functions, particularly those that inhibit CYPs causing increased Cmax (FDA 2012b), such as ketoconazole (Rodriguez and Acosta 1996), the likelihood of developing fatal liver injury could be increased. Of course, as regorafenib is used for cancer patients who have exhausted conventional treatment options, the liver risks, as well as the mitotoxicity reported here, shall be carefully weighed against its clinical benefits. However, as has been highlighted in a recent commentary, drug hepatotoxicity in patients with advanced cancers shall not be underestimated using a looser criterion (Senior 2013). New strategies to minimize regorafenib induced liver injury are of key importance and the present study provides useful insights into mechanisms that should be considered in such strategies.

In summary, we demonstrated the regorafenib impaired mitochondrial functions, activated AMPK kinase, induced autophagy and caused rat hepatocytes necrosis at clinically relevant concentrations. Our study provides novel insights into the mechanisms of regorafenib induced hepatotoxicity. 


\section{References}

Apostolova, N., Gomez-Sucerquia, L.J., Gortat, A., Blas-Garcia, A. and Esplugues, J.V. 2011. Compromising mitochondrial function with the antiretroviral drug efavirenz induces cell survival-promoting autophagy. Hepatology 54, 1009-1019.

Brand, M.D. and Nicholls, D.G. 2011. Assessing mitochondrial dysfunction in cells. The Biochemical journal 435, 297-312.

Bridges, H.R., Jones, A.J., Pollak, M.N. and Hirst, J. 2014. Effects of metformin and other biguanides on oxidative phosphorylation in mitochondria. The Biochemical journal 462, 475-487. Bruix, J., Tak, W.Y., Gasbarrini, A., Santoro, A., Colombo, M., Lim, H.Y., Mazzaferro, V., Wiest, R., Reig, M., Wagner, A. and Bolondi, L. 2013. Regorafenib as second-line therapy for intermediate or advanced hepatocellular carcinoma: multicentre, open-label, phase II safety study. European journal of cancer 49, 3412-3419. Cervinkova, Z., Lotkova, H., Krivakova, P., Rousar, T., Kucera, O., Tichy, L., Cervinka, M. and Drahota, Z. 2007. Evaluation of mitochondrial function in isolated rat hepatocytes and mitochondria during oxidative stress. Alternatives to laboratory animals : ATLA 35, 353-361. Chan, F.K., Moriwaki, K. and De Rosa, M.J. 2013. Detection of necrosis by release of lactate dehydrogenase activity. Methods in molecular biology 979, 65-70. 
Davis, S.L., Eckhardt, S.G., Messersmith, W.A. and Jimeno, A. 2013. The development of regorafenib and its current and potential future role in cancer therapy. Drugs of today 49, 105115.

Ding, W.X. and Yin, X.M. 2009. Analyzing macroautophagy in hepatocytes and the liver. Methods in enzymology 453, 397-416.

Dykens, J.A. and Will, Y. 2007. The significance of mitochondrial toxicity testing in drug development. Drug discovery today 12, 777-785.

Egan, D.F., Shackelford, D.B., Mihaylova, M.M., Gelino, S., Kohnz, R.A., Mair, W., Vasquez, D.S., Joshi, A., Gwinn, D.M., Taylor, R., Asara, J.M., Fitzpatrick, J., Dillin, A., Viollet, B., Kundu, M., Hansen, M. and Shaw, R.J. 2011. Phosphorylation of ULK1 (hATG1) by AMP-activated protein kinase connects energy sensing to mitophagy. Science 331, 456-461.

EMA. 2013. Stivarga (regorafenib).

FDA. 2012a. Regorafenib.

FDA. 2012b. Stivarga (regorafenib) Tablets: Pharmacology Review(s).

Frezza, C., Cipolat, S. and Scorrano, L. 2007. Organelle isolation: functional mitochondria from mouse liver, muscle and cultured fibroblasts. Nature protocols 2, 287-295.

Gomez-Lechon, M.J., Ponsoda, X., O'Connor, E., Donato, T., Jover, R. and Castell, J.V. 2003. Diclofenac induces apoptosis in hepatocytes. Toxicology in vitro : an international journal published in association with BIBRA 17, 675-680.

Han, D., Matsumaru, K., Rettori, D. and Kaplowitz, N. 2004. Usnic acid-induced necrosis of cultured mouse hepatocytes: inhibition of mitochondrial function and oxidative stress.

Biochemical pharmacology 67, 439-451.

Health-Canada. 2013. Stivarga.

Horina, J.H., Wirnsberger, G.H., Kenner, L., Holzer, H. and Krejs, G.J. 1993. Increased susceptibility for CsA-induced hepatotoxicity in kidney graft recipients with chronic viral hepatitis C. Transplantation 56, 1091-1094.

Imberti, R., Nieminen, A.L., Herman, B. and Lemasters, J.J. 1993. Mitochondrial and glycolytic dysfunction in lethal injury to hepatocytes by t-butylhydroperoxide: protection by fructose, cyclosporin A and trifluoperazine. J Pharmacol Exp Ther 265, 392-400.

Jones, D.P., Lemasters, J.J., Han, D., Boelsterli, U.A. and Kaplowitz, N. 2010. Mechanisms of pathogenesis in drug hepatotoxicity putting the stress on mitochondria. Molecular interventions 10, 98-111.

Kon, K., Kim, J.S., Jaeschke, H. and Lemasters, J.J. 2004. Mitochondrial permeability transition in acetaminophen-induced necrosis and apoptosis of cultured mouse hepatocytes. Hepatology 40 , 1170-1179.

Leone, A., Di Gennaro, E., Bruzzese, F., Avallone, A. and Budillon, A. 2014. New perspective for an old antidiabetic drug: metformin as anticancer agent. Cancer treatment and research 159, 355-376.

Lim, C.T., Lolli, F., Thomas, J.D., Kola, B. and Korbonits, M. 2012. Measurement of AMP-activated protein kinase activity and expression in response to ghrelin. Methods in enzymology 514, $271-$ 287.

Malhi, H., Gores, G.J. and Lemasters, J.J. 2006. Apoptosis and necrosis in the liver: a tale of two deaths? Hepatology 43, S31-44.

Malhi, H., Guicciardi, M.E. and Gores, G.J. 2010. Hepatocyte death: a clear and present danger. Physiological reviews 90, 1165-1194.

Masubuchi, Y., Yamada, S. and Horie, T. 2000. Possible mechanism of hepatocyte injury induced by diphenylamine and its structurally related nonsteroidal anti-inflammatory drugs. The Journal of pharmacology and experimental therapeutics 292, 982-987. 

mechanism underlying acetaminophen-induced hepatotoxicity in humans and mice involves mitochondrial damage and nuclear DNA fragmentation. The Journal of clinical investigation 122, 1574-1583.

Meyers, L.L., Beierschmitt, W.P., Khairallah, E.A. and Cohen, S.D. 1988. Acetaminophen-induced inhibition of hepatic mitochondrial respiration in mice. Toxicology and applied pharmacology 93 , 378-387.

Nagai, H., Matsumaru, K., Feng, G. and Kaplowitz, N. 2002. Reduced glutathione depletion causes necrosis and sensitization to tumor necrosis factor-alpha-induced apoptosis in cultured mouse hepatocytes. Hepatology 36, 55-64.

Ni, H.M., Bockus, A., Boggess, N., Jaeschke, H. and Ding, W.X. 2012. Activation of autophagy protects against acetaminophen-induced hepatotoxicity. Hepatology 55, 222-232.

Nieminen, A.L., Saylor, A.K., Herman, B. and Lemasters, J.J. 1994. ATP depletion rather than mitochondrial depolarization mediates hepatocyte killing after metabolic inhibition. The American journal of physiology 267, C67-74.

Okuda, T., Norioka, M., Shitara, Y. and Horie, T. 2010. Multiple mechanisms underlying troglitazone-induced mitochondrial permeability transition. Toxicology and applied pharmacology 248, 242-248.

Pernelle, K., Le Guevel, R., Glaise, D., Stasio, C.G., Le Charpentier, T., Bouaita, B., Corlu, A. and Guguen-Guillouzo, C. 2011. Automated detection of hepatotoxic compounds in human hepatocytes using HepaRG cells and image-based analysis of mitochondrial dysfunction with JC1 dye. Toxicology and applied pharmacology 254, 256-266.

Pernicova, I. and Korbonits, M. 2014. Metformin--mode of action and clinical implications for diabetes and cancer. Nature reviews. Endocrinology 10, 143-156.

Pessayre, D., Fromenty, B., Berson, A., Robin, M.A., Letteron, P., Moreau, R. and Mansouri, A. 2012. Central role of mitochondria in drug-induced liver injury. Drug metabolism reviews 44, 3487.

Petronilli, V., Miotto, G., Canton, M., Brini, M., Colonna, R., Bernardi, P. and Di Lisa, F. 1999. Transient and long-lasting openings of the mitochondrial permeability transition pore can be monitored directly in intact cells by changes in mitochondrial calcein fluorescence. Biophysical journal 76, 725-734.

Porceddu, M., Buron, N., Roussel, C., Labbe, G., Fromenty, B. and Borgne-Sanchez, A. 2012. Prediction of liver injury induced by chemicals in human with a multiparametric assay on isolated mouse liver mitochondria. Toxicological sciences : an official journal of the Society of Toxicology 129, 332-345.

Reid, A.B., Kurten, R.C., McCullough, S.S., Brock, R.W. and Hinson, J.A. 2005. Mechanisms of acetaminophen-induced hepatotoxicity: role of oxidative stress and mitochondrial permeability transition in freshly isolated mouse hepatocytes. The Journal of pharmacology and experimental therapeutics 312, 509-516.

Rodriguez-Enriquez, S., He, L. and Lemasters, J.J. 2004. Role of mitochondrial permeability transition pores in mitochondrial autophagy. The international journal of biochemistry \& cell biology 36, 2463-2472.

Rodriguez, R.J. and Acosta, D., Jr. 1996. Inhibition of mitochondrial function in isolated rate liver mitochondria by azole antifungals. Journal of biochemical toxicology 11, 127-131. Saberi, B., Ybanez, M.D., Johnson, H.S., Gaarde, W.A., Han, D. and Kaplowitz, N. 2014. Protein kinase $\mathrm{C}(\mathrm{PKC})$ participates in acetaminophen hepatotoxicity through c-jun- $\mathrm{N}$-terminal kinase (JNK)-dependent and -independent signaling pathways. Hepatology 59, 1543-1554. 

serious drug-induced hepatotoxicity in special groups of trial subjects. Pharmacoepidemiology and drug safety $22,579-582$.

Shackelford, D.B. and Shaw, R.J. 2009. The LKB1-AMPK pathway: metabolism and growth control in tumour suppression. Nature reviews. Cancer 9, 563-575.

Shah, R.R., Morganroth, J. and Shah, D.R. 2013. Hepatotoxicity of tyrosine kinase inhibitors: clinical and regulatory perspectives. Drug safety : an international journal of medical toxicology and drug experience 36, 491-503.

Shi, Q., Yang, X., Greenhaw, J. and Salminen, W.F. 2011. Hepatic cytochrome P450s attenuate the cytotoxicity induced by leflunomide and its active metabolite A77 1726 in primary cultured rat hepatocytes. Toxicological sciences : an official journal of the Society of Toxicology 122, 579586.

Sonko, B.J., Schmitt, T.C., Guo, L., Shi, Q., Boros, L.G., Leakey, J.E. and Beger, R.D. 2011. Assessment of usnic acid toxicity in rat primary hepatocytes using (1)(3)C isotopomer distribution analysis of lactate, glutamate and glucose. Food and chemical toxicology : an international journal published for the British Industrial Biological Research Association 49, 2968-2974.

Starkov, A.A., Bloch, D.A., Chernyak, B.V., Dedukhova, V.I., Mansurova, S.E., Severina, II, Simonyan, R.A., Vygodina, T.V. and Skulachev, V.P. 1997. 6-Ketocholestanol is a recoupler for mitochondria, chromatophores and cytochrome oxidase proteoliposomes. Biochimica et biophysica acta 1318, 159-172.

Sunakawa, Y., Furuse, J., Okusaka, T., Ikeda, M., Nagashima, F., Ueno, H., Mitsunaga, S., Hashizume, K., Ito, Y. and Sasaki, Y. 2013. Erratum to: Regorafenib in Japanese patients with solid tumors: phase I study of safety, efficacy, and pharmacokinetics. Investigational new drugs. Viollet, B., Guigas, B., Leclerc, J., Hebrard, S., Lantier, L., Mounier, R., Andreelli, F. and Foretz, M. 2009. AMP-activated protein kinase in the regulation of hepatic energy metabolism: from physiology to therapeutic perspectives. Acta physiologica 196, 81-98.

Waldmeier, P.C., Feldtrauer, J.J., Qian, T. and Lemasters, J.J. 2002. Inhibition of the mitochondrial permeability transition by the nonimmunosuppressive cyclosporin derivative NIM811. Molecular pharmacology 62, 22-29.

Will, Y., Dykens, J.A., Nadanaciva, S., Hirakawa, B., Jamieson, J., Marroquin, L.D., Hynes, J., Patyna, S. and Jessen, B.A. 2008. Effect of the multitargeted tyrosine kinase inhibitors imatinib, dasatinib, sunitinib, and sorafenib on mitochondrial function in isolated rat heart mitochondria and H9c2 cells. Toxicological sciences : an official journal of the Society of Toxicology 106, 153161. 


\section{Figure legend}

Fig. 1 Effects of regorafenib on mitochondrial respiration and calcium overload-induced swelling. Freshly isolated rat liver mitochondria $(1 \mathrm{mg} / \mathrm{ml}$ protein) were treated by regorafenib $(1,2.5,5$ and $10 \mu \mathrm{M})$ with $0.1 \%$ DMSO as a control, and then oxygen consumption measured after 3 min. $\mathrm{CCCP}(0.3 \mu \mathrm{M})$ was used as a control ( $\mathrm{A}$ and $\mathrm{B})$. In $\mathrm{C}$ and $\mathrm{D}$, oligomycin $\mathrm{A}(2 \mu \mathrm{g} / \mathrm{ml})$ was added to the mitochondria prior to the addition of substrates (glutamate/malate or succinate), and then regorafenib was added, which was followed by $200 \mu \mathrm{M} \mathrm{KC}$, and oxygen consumption was measured 3 min after each treatment. No ADP was added for these experiments. In panel $\mathrm{E}$ and $\mathrm{F}$, regorafenib was used at 1, 2.5, 5, 10 and $15 \mu \mathrm{M}$. In panel E, $1 \mathrm{mM}$ $\mathrm{K}_{2} \mathrm{HPO}_{4}$ and $25 \mu \mathrm{M} \mathrm{CaCl}_{2}$ were used to induce mitochondrial swelling before the absorbance at $535 \mathrm{~nm}$ was recorded for $30 \mathrm{~min}$. In panel F, $1 \mu \mathrm{M}$ CsA was added for $1 \mathrm{~min}$ prior to regorafenib treatment. Data are mean $\pm \mathrm{SD}, \mathrm{n}=3 . * p<0.05$, compared to $0 \mu \mathrm{M}$ regorafenib (that is, $0.1 \%$ to $0.3 \% \mathrm{DMSO}$ ) treated mitochondria; \# $p<0.05$, compared to the corresponding samples treated by $\mathrm{KC}$.

Fig. 2 Effects of regorafenib on hepatocyte oxygen consumption, MMP and MPT. Primary rat hepatocytes were treated with regorafenib $(0,5,7.5,10$, and $15 \mu \mathrm{M})$ alone or together with $\mathrm{KC}(100 \mu \mathrm{M})$ for $1 \mathrm{~h}$, and then cells trypsinized to measure mitochondrial oxygen consumption (A and B). In Fig. C and D, hepatocytes were treated by regorafenib $(0,2.5,5,7.5,10$, and $15 \mu \mathrm{M})$ with or without $\mathrm{KC}$ for $1 \mathrm{~h}$, and then stained with $10 \mu \mathrm{M} \mathrm{JC}-1$ for $15 \mathrm{~min}$. Cells were collected by trypsinization. A small fraction was used for microscopic observation (C), and $50 \mu \mathrm{l}$ cell suspensions were used to measure red and green fluorescence, whose ratio was presented in panel D. In panel E, hepatocytes treated for $3 \mathrm{~h}$ were stained with calcein AM to detect MPT using a flow cytometer, and the corresponding results from three experiments were presented in panel $\mathrm{F}$. The quantitative data are mean $\pm \mathrm{SD}, \mathrm{n}=3$. ${ }^{*} p<0.05$, compared to vehicle $(0.2 \%$ DMSO) treated hepatocytes; \# $p<0.05$, compared to the corresponding samples treated by KC.

Fig. 3 Effects of regorafenib on hepatocyte cellular ATP levels, LDH release, apoptosis, and caspase activity. Primary rat hepatocytes were treated with regorafenib alone or together with $\mathrm{KC}$ for 1 to $24 \mathrm{~h}$, and 
cellular ATP and LDH were measured at 1, 3, 7 and $24 \mathrm{~h}$ (panel A to D). At $7 \mathrm{~h}$, hepatocytes were stained by Hoechst 33258 to visualize nuclear changes that reflect apoptosis (panel E), whose quantitative data were shown in Fig. F. The hepatocyte apoptosis was also measured by caspase 3/7 activities (panel G). Diclofenac was used as a positive control for apoptosis detection. The quantitative data are mean $\pm S D, n=3$. $* p<0.05$, compared to vehicle $(0.2 \%$ DMSO) treated hepatocytes; \# $p<0.05$, compared to the corresponding samples treated by KC.

Fig. 4 Effects of fructose, oligomycin A, cyclosporine A (CsA), and trifluoperazine (TFP) on regorafenib induced hepatocyte death. Primary hepatocytes were treated with regorafenib in the absence or presence of fructose $(15 \mathrm{mM})$, or fructose plus oligomycin $\mathrm{A}(0.6 \mathrm{ng} / \mathrm{ml})$, or cyclosporine $\mathrm{A}(\mathrm{CsA} ; 0.5 \mu \mathrm{M})$ plus trifluoperazine (TFP; $2.5 \mu \mathrm{M}$ ), and $\mathrm{LDH}$ release was measured at $7 \mathrm{~h}$ and $24 \mathrm{~h}$. The data are mean $\pm \mathrm{SD}$, $\mathrm{n}=3 . * p<0.05$, compared to vehicle $(0.1 \%$ or $0.2 \%$ DMSO) treated hepatocytes; $\# p<0.05$, compared to the corresponding cells treated by regorafenib alone.

Fig. 5 AMPK activation and effects of Compound $\mathrm{C}$ in regorafenib treated hepatocytes. Primary rat hepatocytes were treated by regorafenib for $1 \mathrm{~h}$ to $7 \mathrm{~h}$, and then AMPK activation was detected by Western blot (panel A). p-AMPK level was normalized to beta-actin and the quantitative data were presented in panel B. In panel C and D, LDH release was measured at $7 \mathrm{~h}$ and $24 \mathrm{~h}$ after hepatocytes were treated with regorafenib in the absence or presence of $10 \mu \mathrm{M}$ Compound $\mathrm{C}$. The effect of KC on AMPK activation is shown in panel $\mathrm{E}$ and $\mathrm{F}$. All quantitative data are mean $\pm \mathrm{SD}, \mathrm{n}=3$. $* p<0.05$, compared to vehicle $(0.2 \%$ DMSO) treated hepatocytes; \# $p<0.05$, compared to the corresponding cells treated by regorafenib alone.

Fig. 6 LC3-II accumulation and effects of autophagy modulators in regorafenib treated hepatocytes. Primary rat hepatocytes were treated by regorafenib for $1 \mathrm{~h}$ to $7 \mathrm{~h}$, and then LC3-II levels were detected by Western blot (panel A). LC3-II levels were normalized to beta-actin and the quantitative data were presented in panel B. In panel C and D, LDH release was measured at $7 \mathrm{~h}$ and $24 \mathrm{~h}$ after hepatocytes were treated with regorafenib in the absence or presence of $5 \mu \mathrm{M}$ rapamycin (Rap) or $15 \mu \mathrm{M}$ chloroquine (CQ). The quantitative data are mean $\pm \mathrm{SD}, \mathrm{n}=3 .{ }^{*} p<0.05$, compared to vehicle $(0.1$ to $0.2 \% \mathrm{DMSO})$ treated hepatocytes; \# $p<0.05$, compared to the corresponding cells treated with regorafenib alone. 
Fig. 7 A schematic diagram of major findings in the present study. 1. KC, 6-ketocholestanol, a recoupler; 2. $\mathrm{C}+\mathrm{T}$, cyclosporin $\mathrm{A}(\mathrm{Cs} \mathrm{A})$ and trifluoperazine (TFP), MPT blockers; 3. $\mathrm{F}+\mathrm{O}$, fructose and oligomycin $\mathrm{A}$, a glycolysis enhancer and a mitochondrial ATPase synthase inhibitor, respectively; 4. CC, Compound C, an AMPK inhibitor; 5. CQ, chloroquine, an autophagy inhibitor; 6. RAP, rapamycin, an autophagy inducer. Red and green arrows indicate pro-death and pro-survival pathways, respectively. 

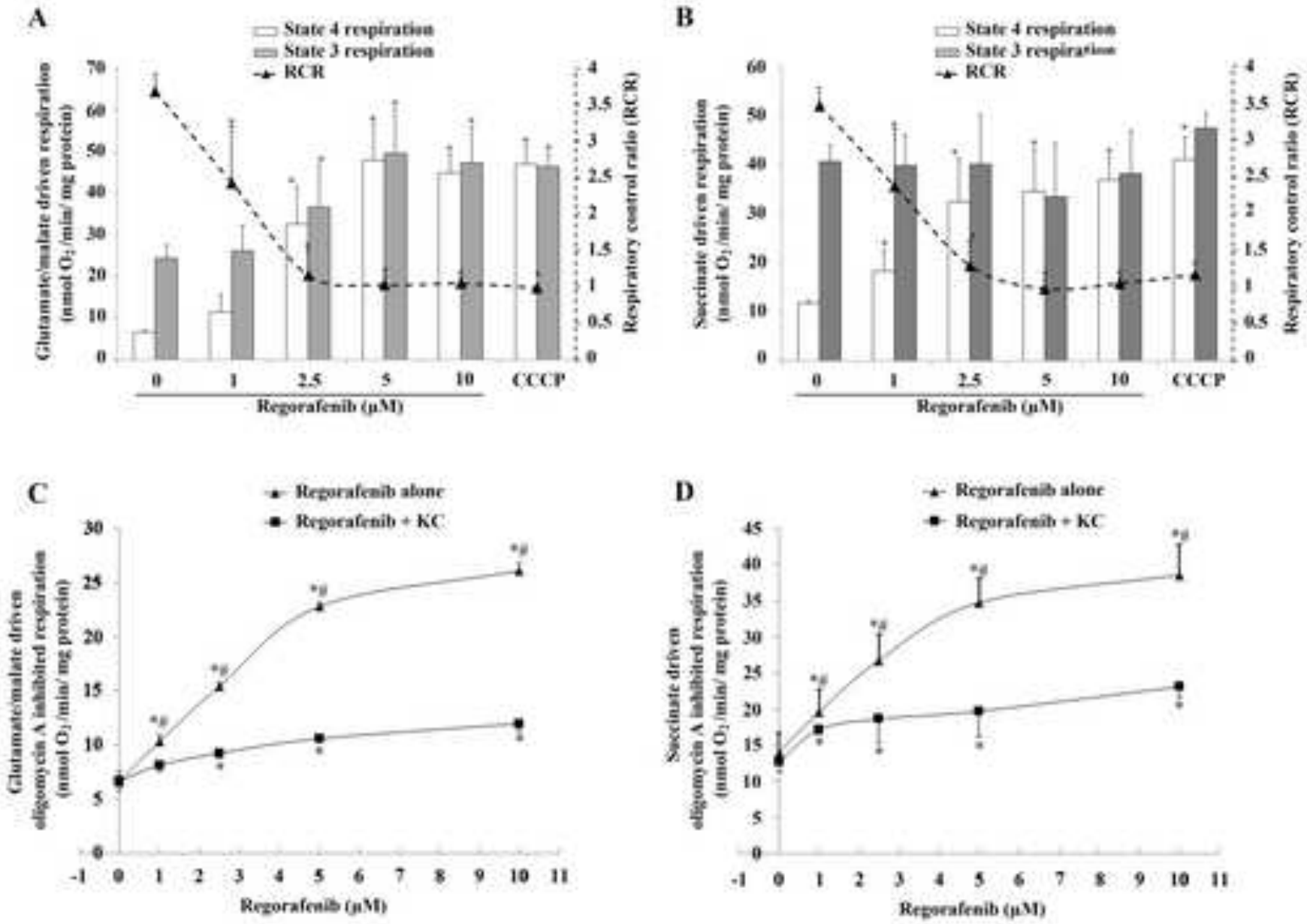

E

F
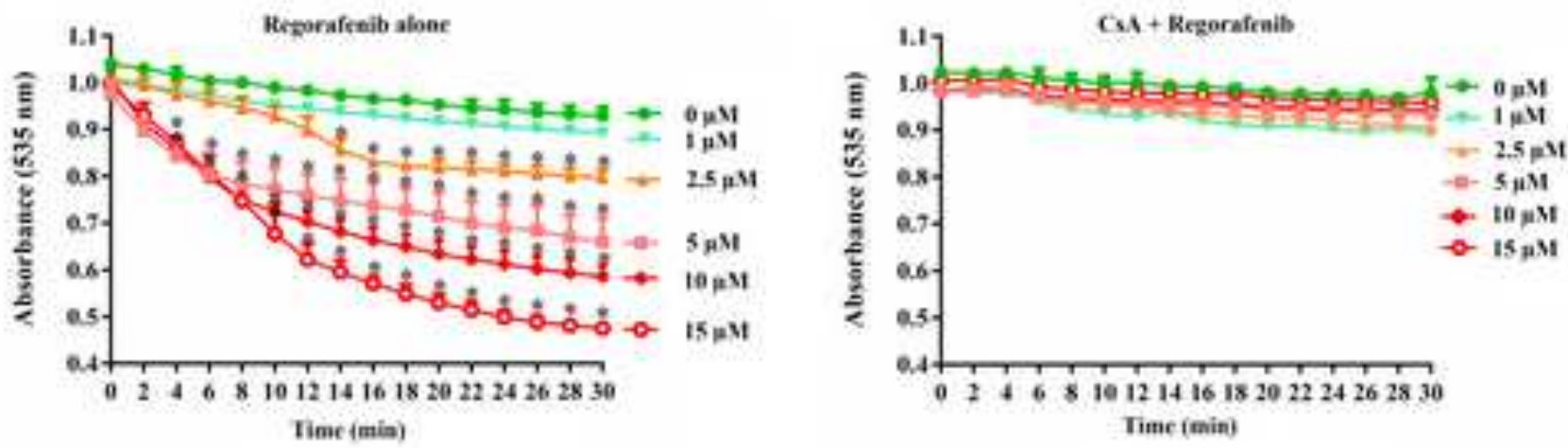

Figure 1 
A

$$
\begin{aligned}
& \text { Rezoratenibabue Rezorafenih }+\mathbf{K C} \\
& \begin{array}{ll}
\square \text { State } 4 & =\text { Statr } 4 \\
\text { - State } 3 & =\text { State } 3 \\
- \text { RCR } & - \text { RCR }
\end{array}
\end{aligned}
$$

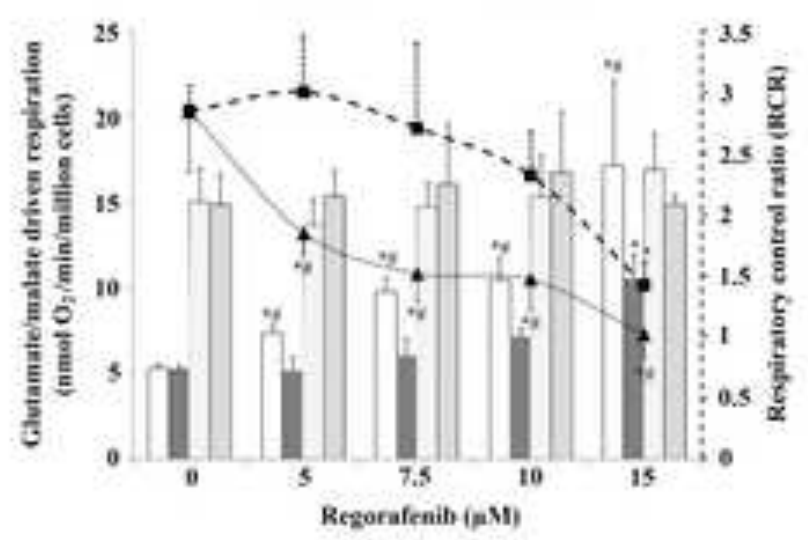

B

$$
\begin{aligned}
& \text { Regerafeaitialone Regorafenib + KC } \\
& \square \text { State } 4 \text { - State } 4 \\
& \text { - State } 3=\text { State } 3 \\
& \text { - MCE }-\mathrm{RCH}
\end{aligned}
$$

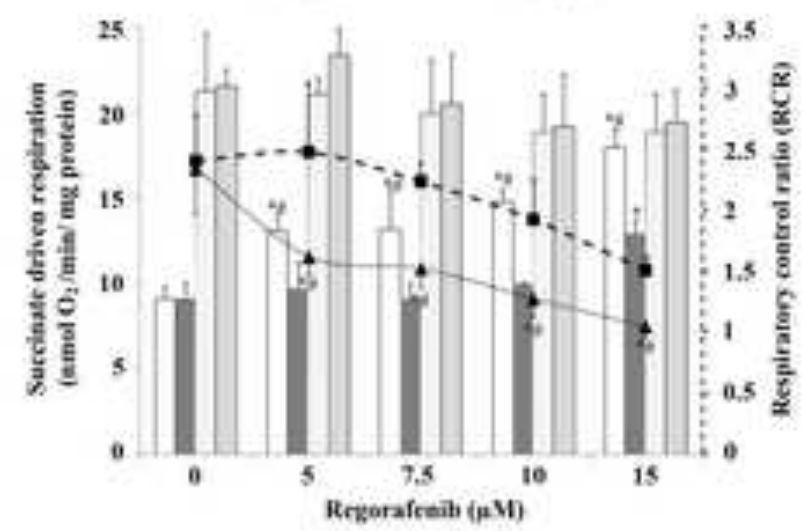

C

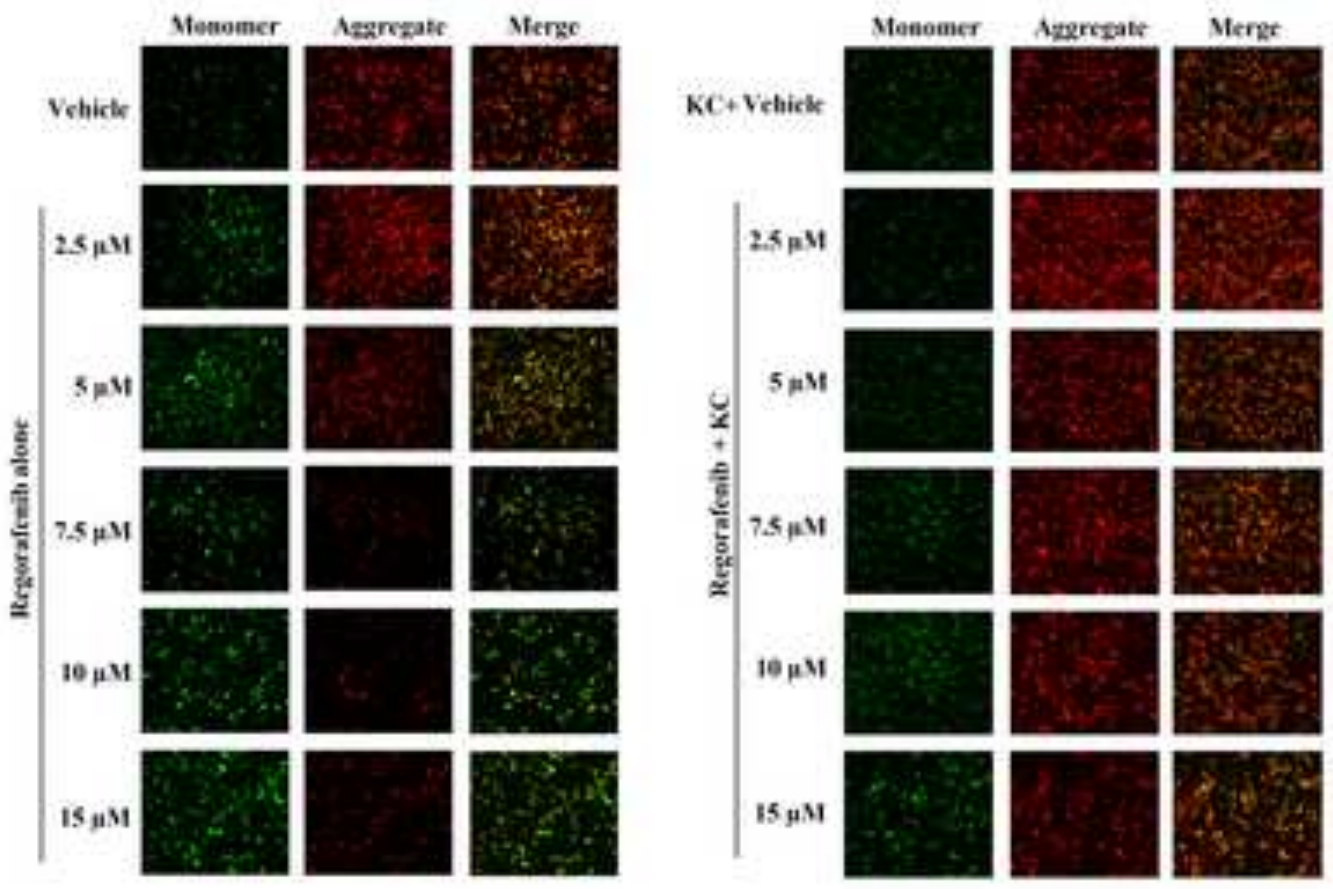

D

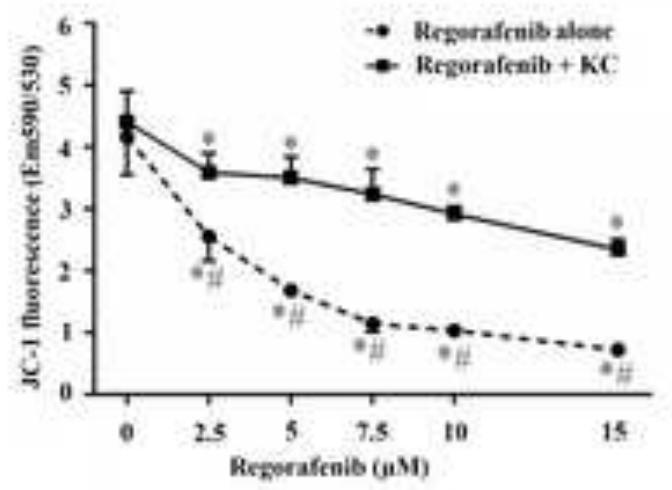

Figure 2A-D 
2E
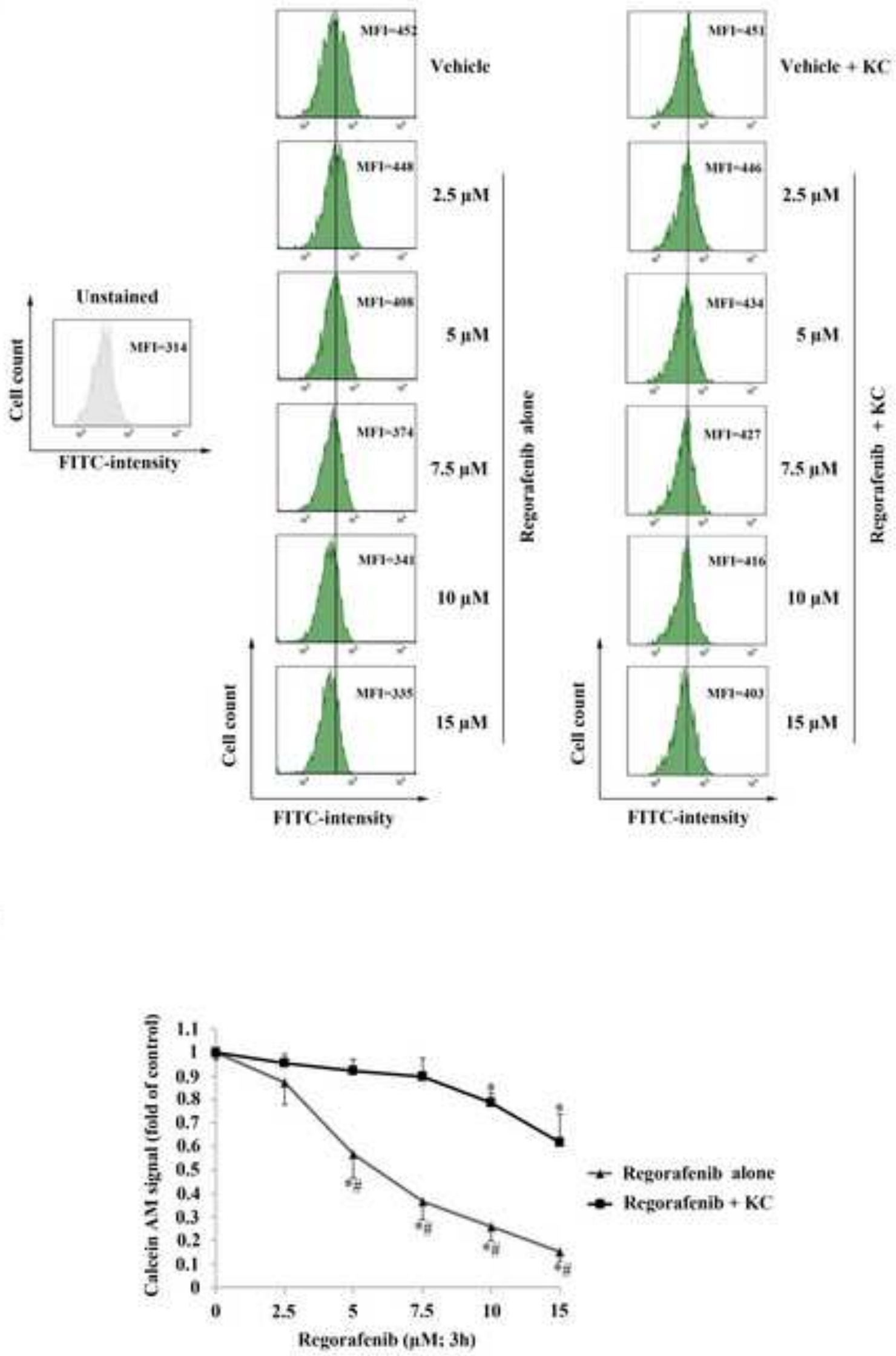

Figure 2E\&F 
A

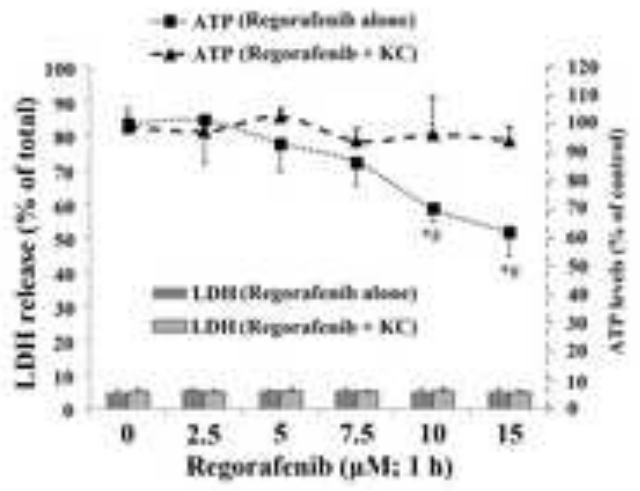

C

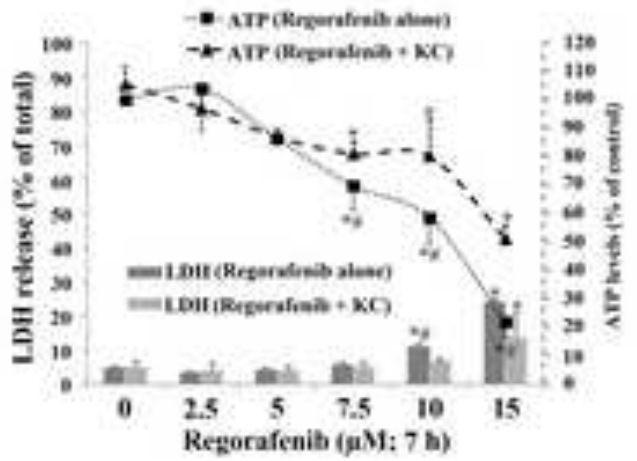

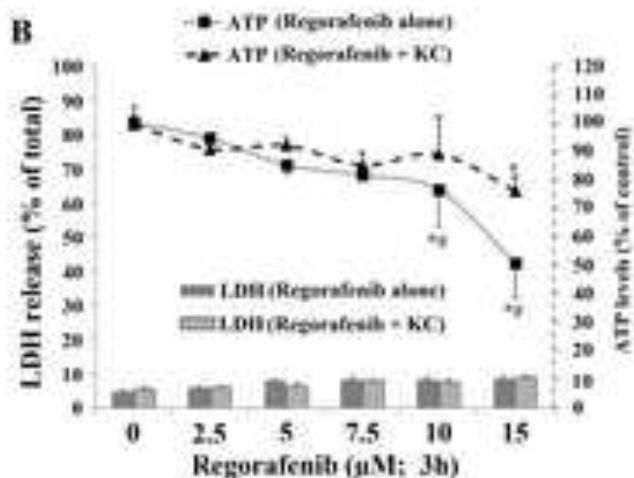

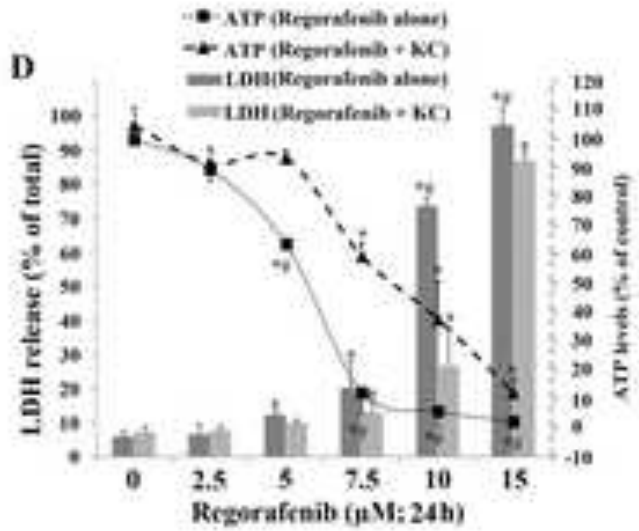

E

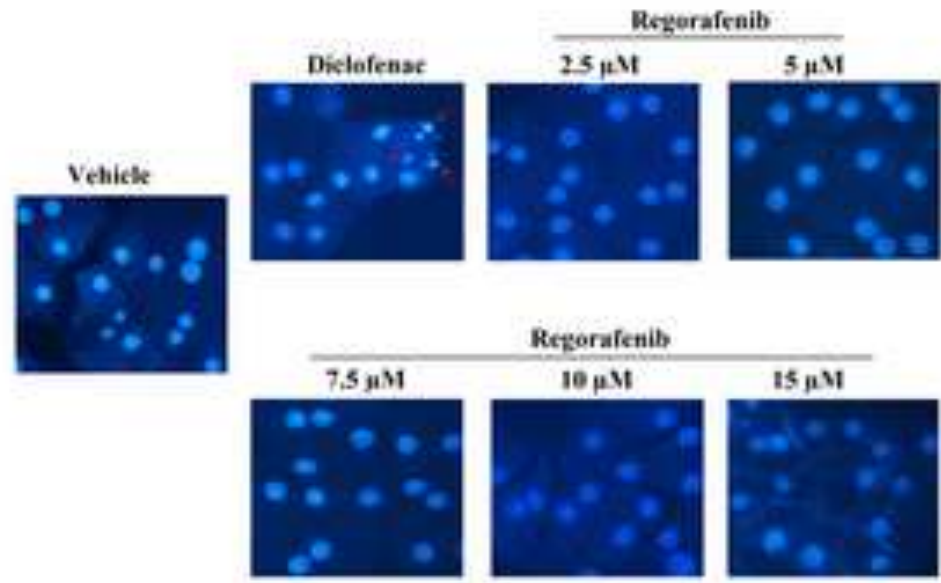

F

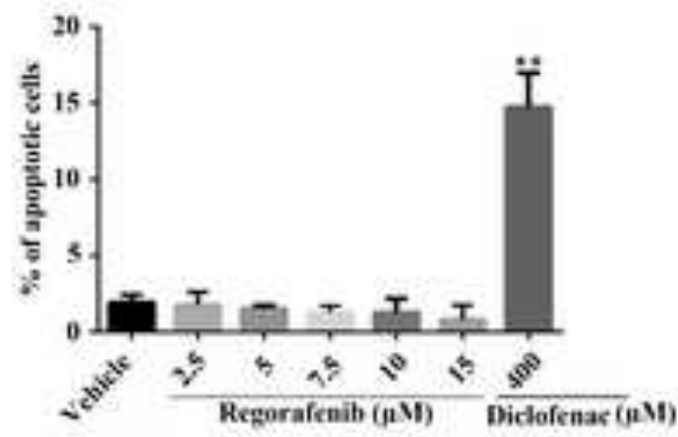

G.

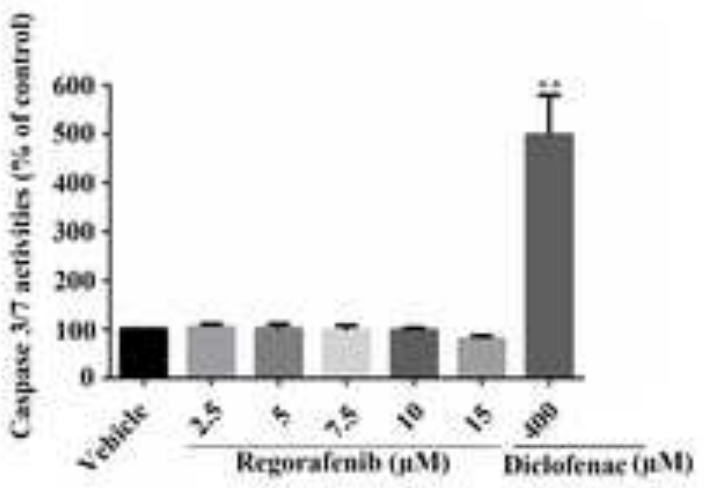


A

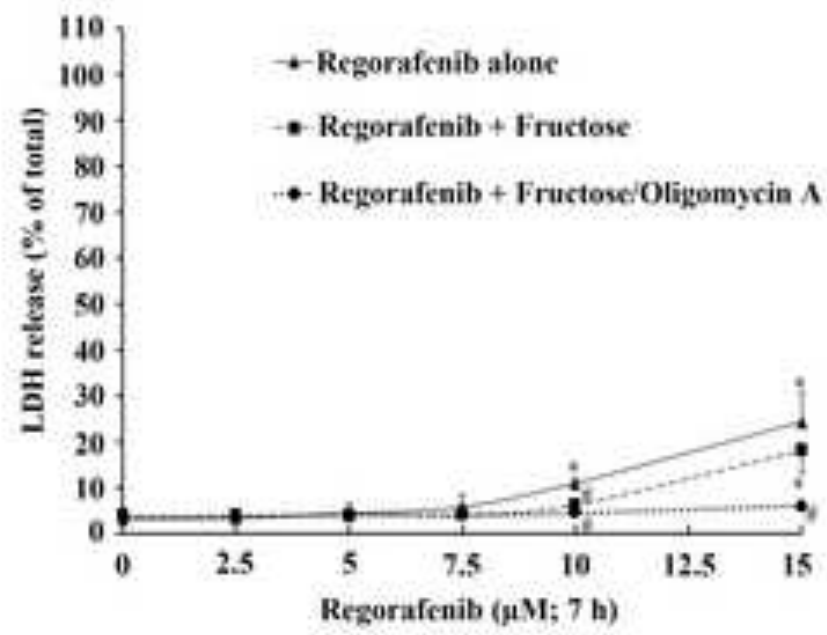

C

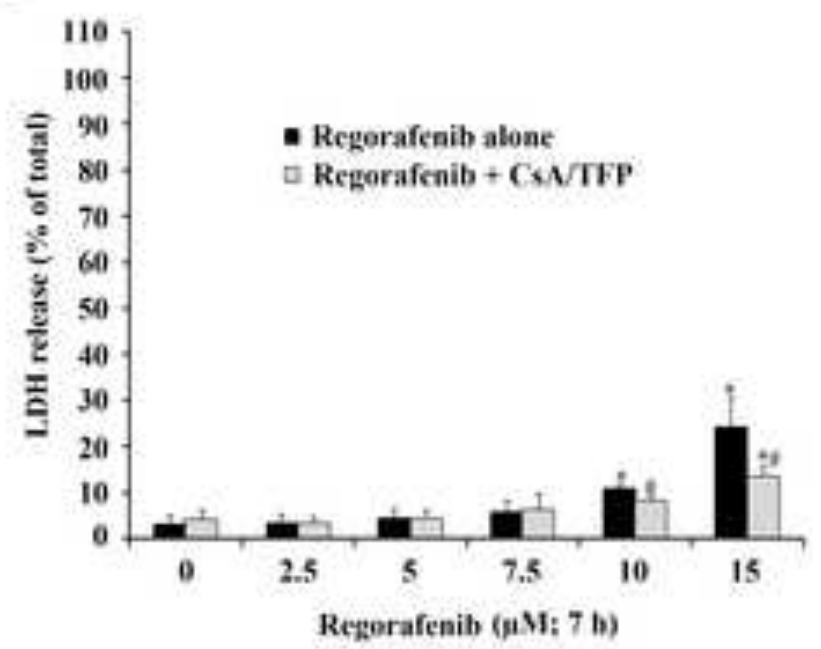

B

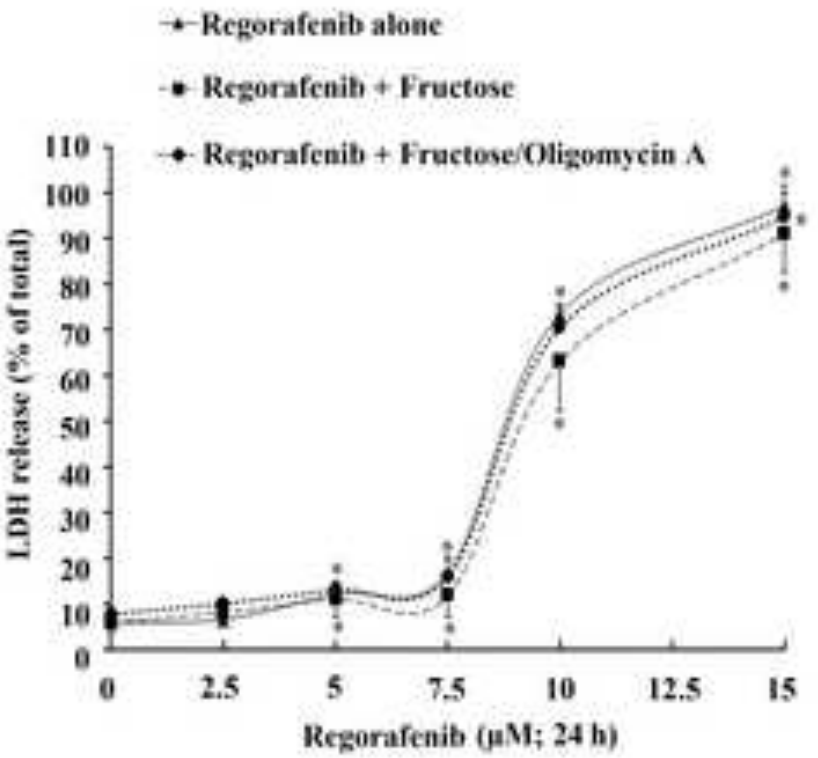

D

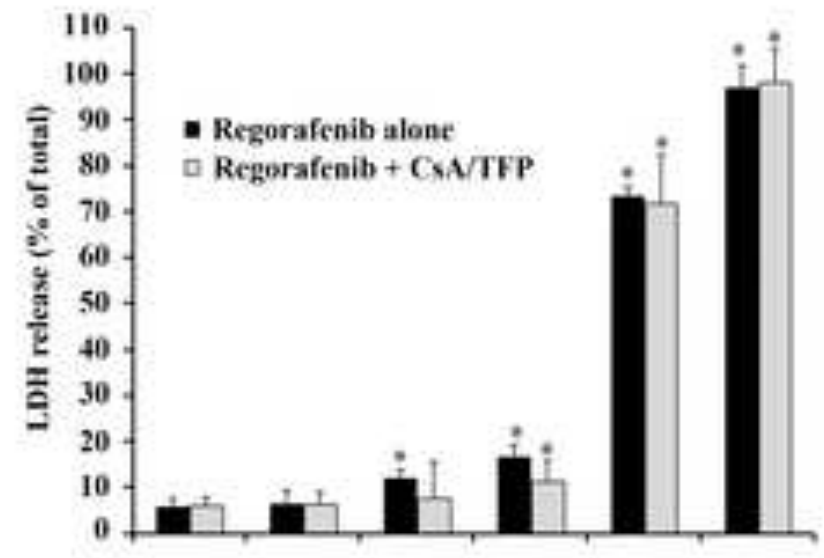

Regorafenib ( $\mu \mathrm{MI}: 24 \mathrm{~h}$ )

Figure 4 


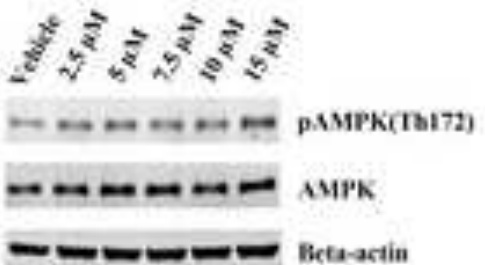

Regorafenib ( i b)

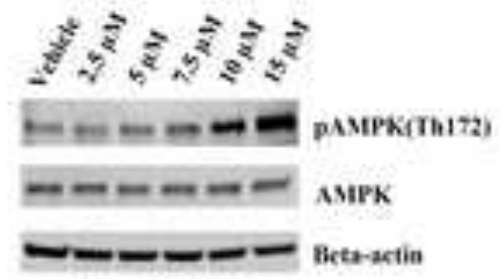

Nergorafraib (s b)

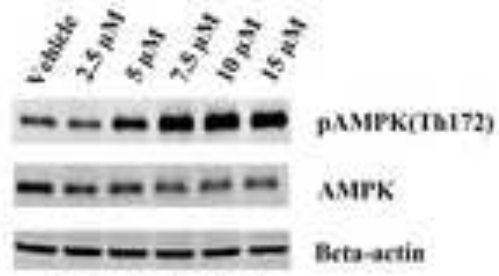

Regorafcuib (t h)
B

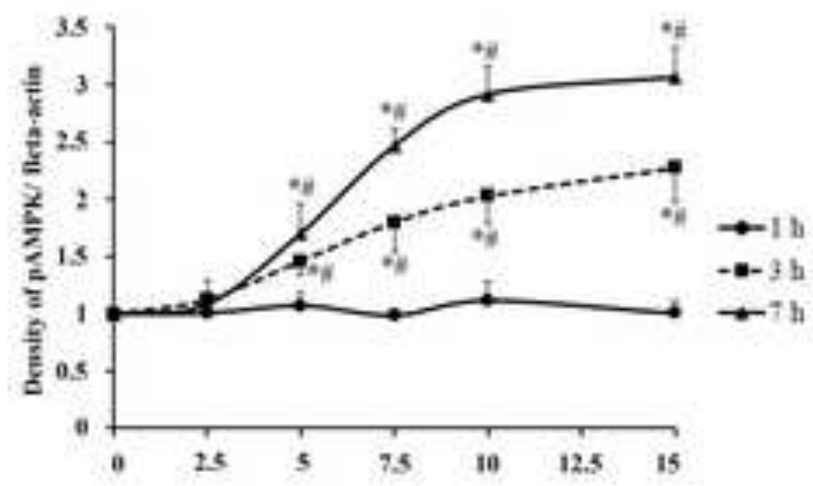

D

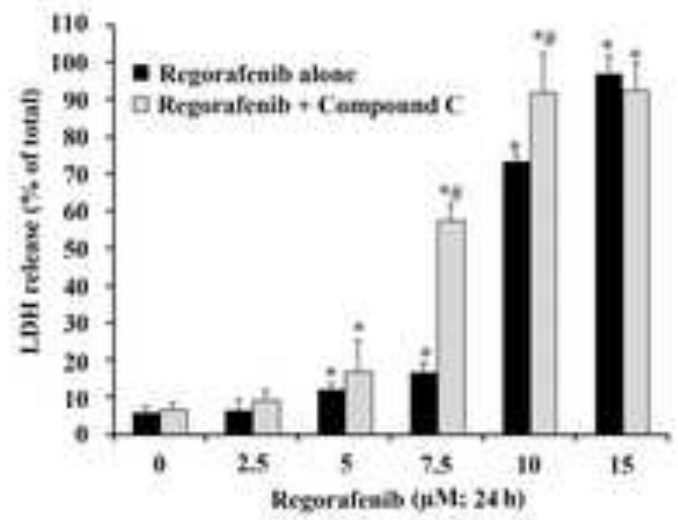

F

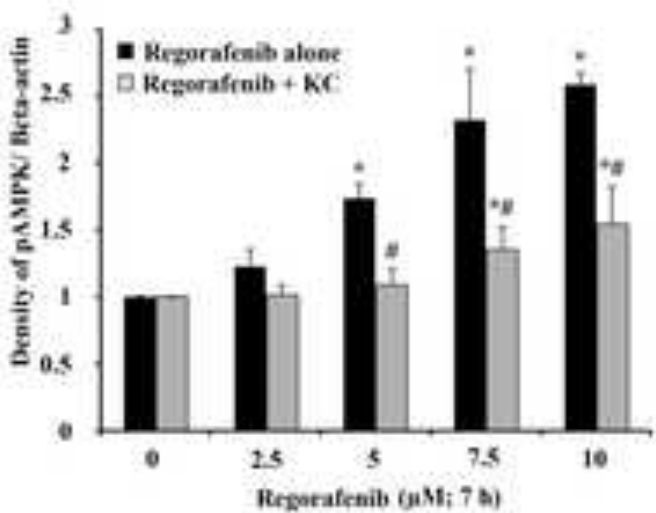

C

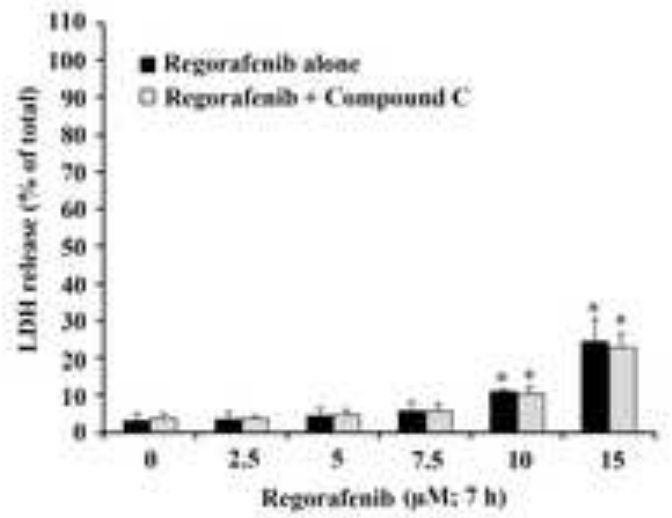

E.

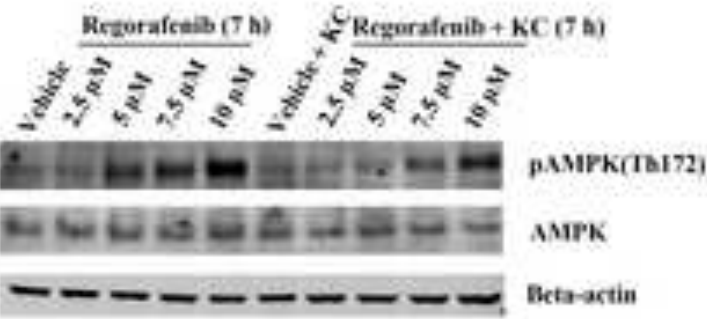


A

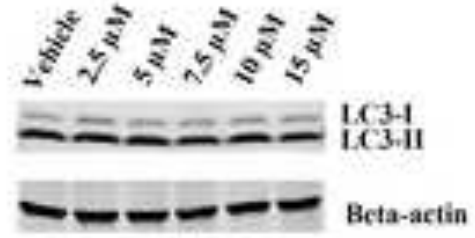

Regorafenib (1 h)

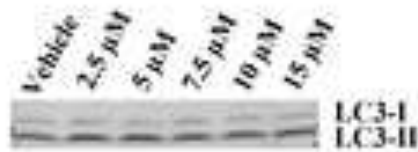

Beta-actin

Rezorafenib (3 h)

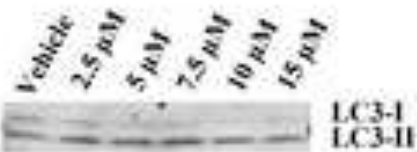

LC3-II

Beta-actin

Regorafeaib ( 7 b)
B

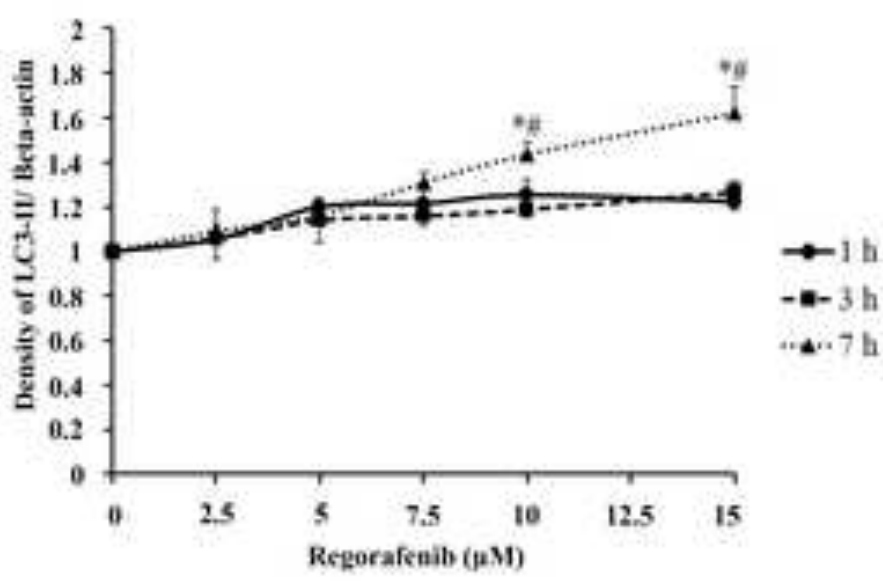

D

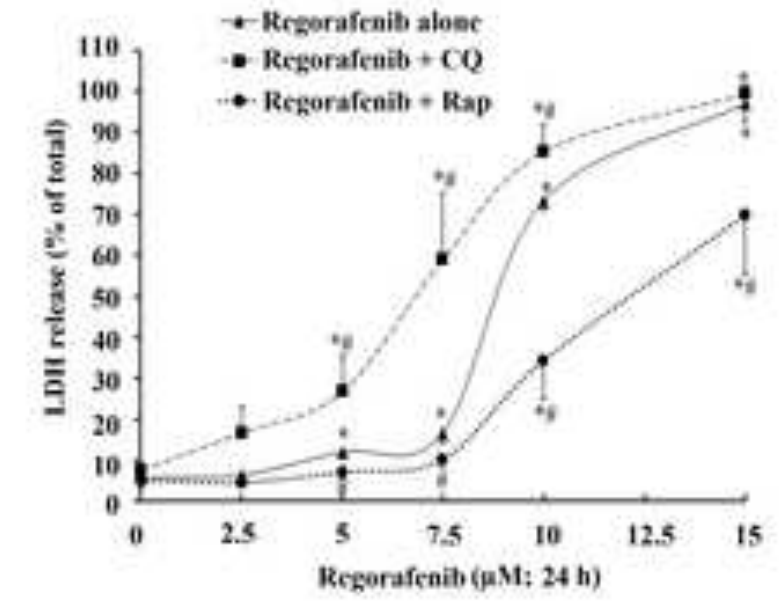

Figure 6
C

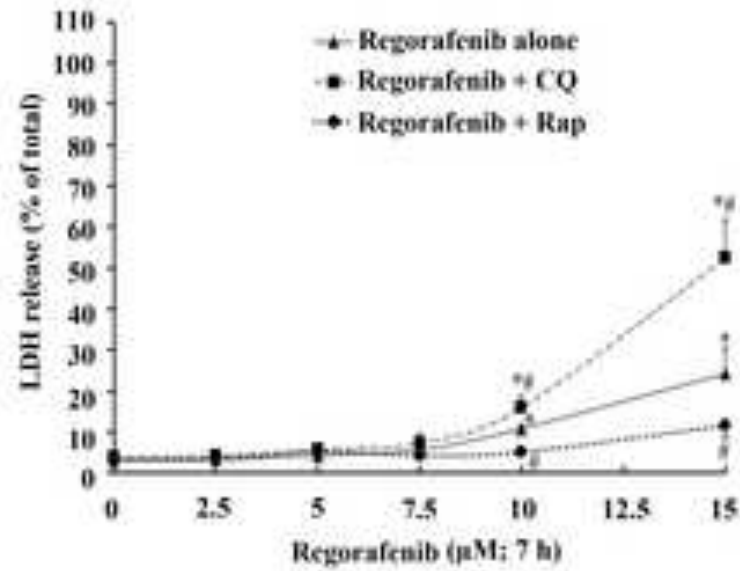




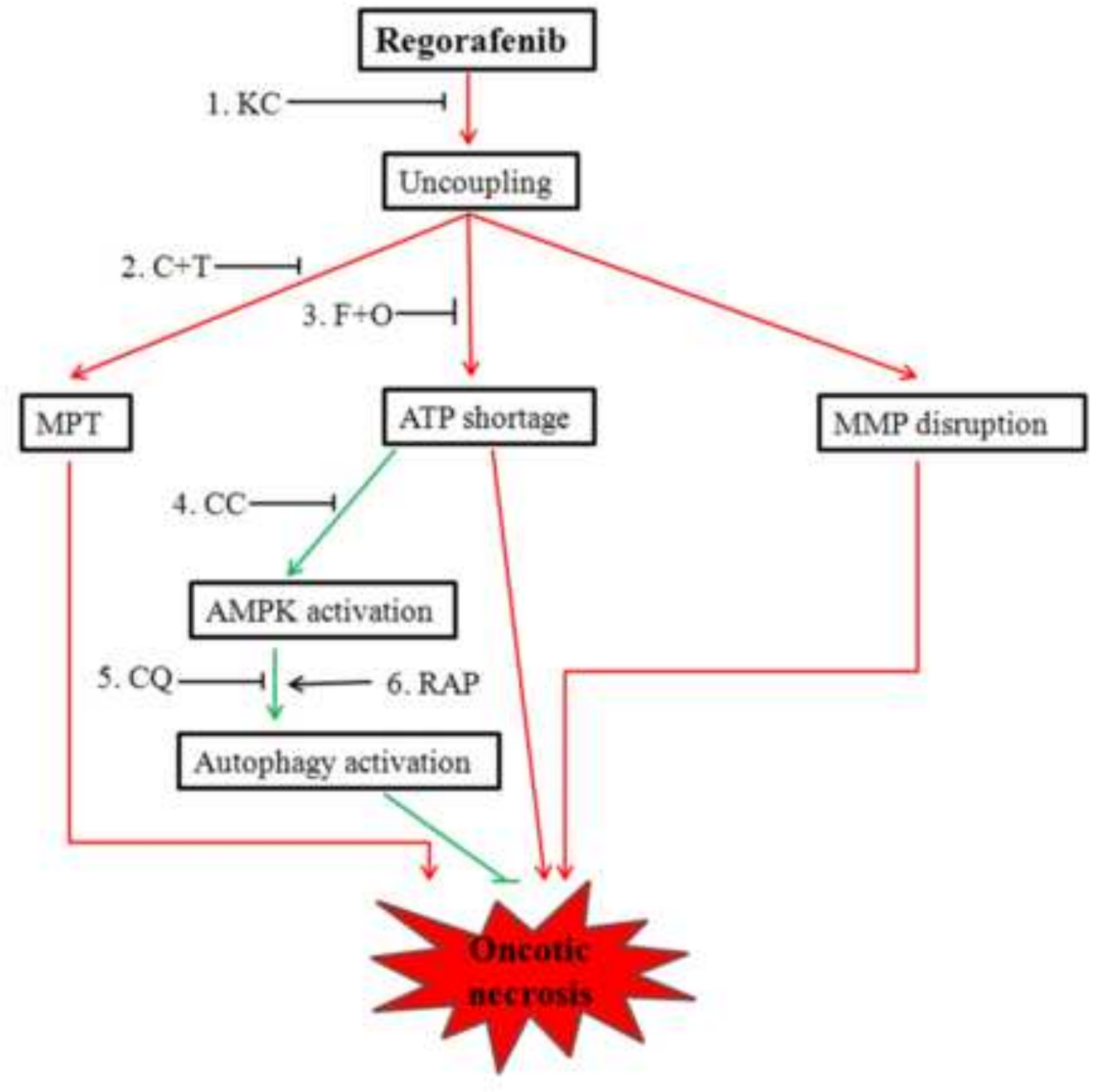

